\title{
The initial steps of ovarian cancer cell metastasis are mediated by MMP-2 cleavage of vitronectin and fibronectin
}

\author{
Hilary A. Kenny, ${ }^{1}$ Swayamjot Kaur, ${ }^{1}$ Lisa M. Coussens, ${ }^{2}$ and Ernst Lengyel ${ }^{1,3}$ \\ ${ }^{1}$ Department of Obstetrics and Gynecology, Section of Gynecologic Oncology, and Gordon Center for Integrative Science, \\ University of Chicago, Chicago, Illinois, USA. ${ }^{2}$ Department of Pathology and Comprehensive Cancer Center, UCSF, San Francisco, California, USA. \\ ${ }^{3}$ Committee on Cancer Biology, University of Chicago, Chicago, Illinois, USA.
}

\begin{abstract}
Most patients (80\%) with ovarian cancer (OvCa) present with metastatic disease. Attachment of OvCa cells to peritoneum and omentum represents the first rate-limiting step for metastatic spread. Therefore, identifying factors regulating cell attachment in the abdominal cavity is critical to the development of therapeutic agents. We show here that MMP-2 expression was upregulated in OvCa cells upon attachment to their microenvironment. Downregulation of MMP-2 mRNA or pharmacological inhibition of MMP-2 proteolytic function, in both human OvCa primary cells and cell lines, reduced attachment of OvCa cells to a 3D organotypic model of metastatic OvCa, full human omentum or peritoneum, and in vivo to mouse peritoneum and omentum. Absence of MMP-2 in the host did not alter OvCa adhesion, as determined utilizing mice harboring homozygous null mutations in either the Mmp2 or $M m p 9$ genes. Conversely, adhesion induced upregulation of MMP-2 mRNA in OvCa cells. MMP-2 inhibition in OvCa cells through pharmacological or antibody treatment prior to i.p. dissemination in nude mice significantly decreased tumor growth and metastasis and extended survival. MMP-2 enhanced peritoneal adhesion of OvCa cells through cleavage of ECM proteins fibronectin (FN) and vitronectin $(\mathrm{Vn})$ into small fragments and increased binding of OvCa cells to these FN and $\mathrm{Vn}$ fragments and their receptors, $\alpha_{5} \beta_{1}$ and $\alpha_{v} \beta_{3}$ integrin. These findings indicate that MMP-2 expressed by metastatic OvCa cells functionally regulates their attachment to peritoneal surfaces.
\end{abstract}

\section{Introduction}

Ovarian cancer (OvCa) has the highest mortality rate of all gynecologic tumors and is the fifth leading cause of cancer death among US women (1). It is predominantly confined within the abdominal cavity and, unlike breast, colon, or lung cancer, rarely metastasizes hematogenously. Once an ovarian epithelial cell undergoes neoplastic transformation, it freely disseminates throughout the peritoneal cavity, carried by peritoneal fluid that facilitates attachment to peritoneum and omentum. The omentum is a large fat pad (approximately $12 \times 12 \mathrm{~cm}$ ) located inferior to the stomach and draped over the small bowel. It is the most common metastatic site (80\%) for OvCa cells (2) followed by implants on the abdominal peritoneum. Identification of cofactors regulating OvCa cell attachment to omentum and/or peritoneum would have tremendous clinical utility, by enabling identification of cellular or molecular targets that could be pursued therapeutically and thus, enabling blockade of a critical step necessary for OvCa metastasis within the peritoneal cavity.

A role for MMPs in OvCa development has been postulated based upon the observation that several members of the MMP family are upregulated during OvCa neoplastic progression (3). When MMPs were first characterized (4), it was hypothesized that their major contribution to cancer development was merely to

Nonstandard abbreviations used: APMA, aminophenylmercuric acetate; FACS, fluorescent-activated cell sorting; FN, fibronectin; HPF, human primary fibroblast; HPMC, human primary mesothelial cell; MMPI, MMP inhibitor; MT1, membranetype 1 ; OvCa, ovarian cancer; $\mathrm{Vn}$, vitronectin.

Conflict of interest: The authors have declared that no conflict of interest exists. Citation for this article: J. Clin. Invest. 118:1367-1379 (2008). doi:10.1172/JCI33775 degrade ECM molecules, thereby facilitating cancer cell migration/invasion across tissue boundaries. More recent insights have, however, defined a more complex role for MMPs in cancer. They are now recognized as key regulators of various neoplastic processes by virtue of their ability to mediate differentiation, proliferation, and survival of neoplastic cells (5), release mitogenic growth factors from cell surfaces and from ECM reservoirs, and regulate tumor-associated angiogenesis $(6,7)$. In spite of these revelations, no MMPs have been identified as being absolutely required for neoplastic cell migration/invasion into ectopic tissue compartments in vivo. Based upon their perceived importance as mediators of ECM remodeling, clinical trials assessing efficacy of broad spectrum MMP inhibitors (MMPI) in patients with solid tumors, including non-small cell lung (8), pancreatic (9), gastric (10), and OvCas (11), were undertaken in patients with recurrent, metastatic, chemotherapy-resistant tumors. Unfortunately, none of the MMPI evaluated improved patient survival (12).

Interestingly, several groups using preclinical mouse models of de novo cancer development $(7,13)$ revealed that MMPI efficacy may be best achieved during earlier stages of tumor development, prior to appearance of bulky and/or metastatic disease. In the RIP1-Tag2 model of pancreatic islet carcinogenesis (7), tumor burden was significantly reduced in tumor-prone mice when mice were treated with the MMPI batimastat during early neoplasia, prior to malignant conversion and development of islet adenocarcinomas. Moreover, if tumor-prone mice were treated later in their disease progression, when tumors were already present, there was no significant effect (14). Similarly, growth of OvCa xenografts is significantly diminished if mice are treated with batimastat immediately following tumor cell injection; whereas, if batima- 
A

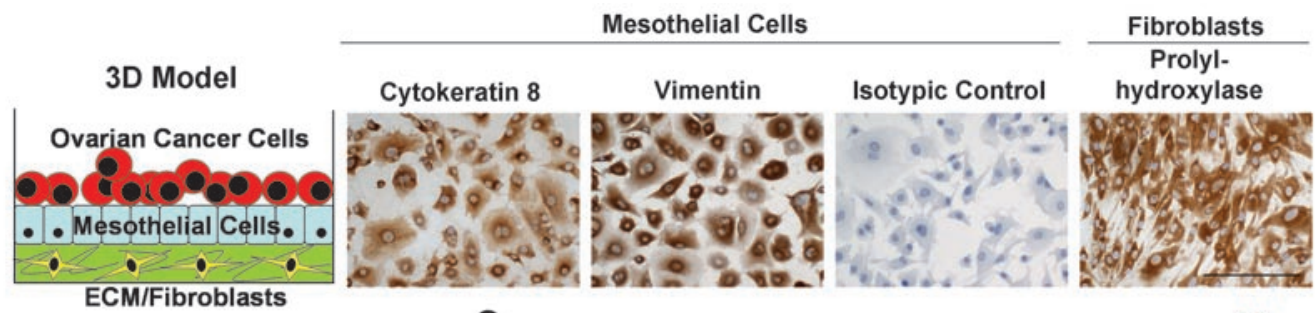

$\mathbf{B}$
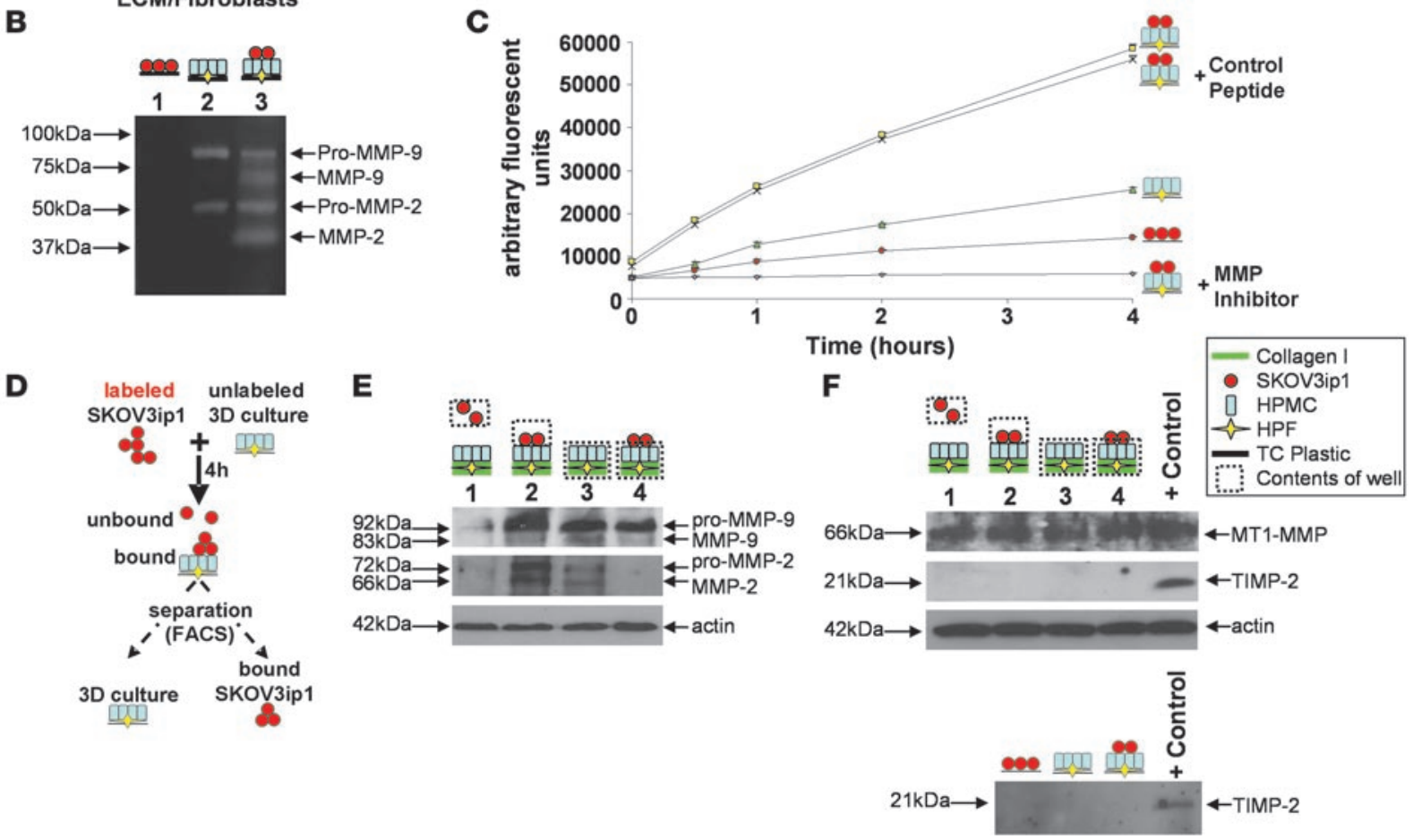

Figure 1

Binding of OvCa cells to an omental 3D culture increases MMP-2/-9 expression and secretion in cancer cells. (A) Concept for a 3D culture to imitate abdominal mesothelium. HPMCs stained with an antibody against cytokeratin 8 , vimentin, or an isotypic specific control antibody. HPFs stained with a prolyl-hydroxylase antibody recognizing human fibroblasts. Scale bar: $50 \mu \mathrm{M}$. (B) Zymogram. Conditioned media from SKOV3ip1 cells (red circles), the 3D culture (composed of HPMCs [blue rectangles], HPFs [yellow diamonds], and collagen I [green rectangles]), or coculture was subjected to gelatin zymography. (C) Gelatinase assay. Cell-associated gelatinolytic activity in the indicated cell populations, with or without an MMP-2/-9 inhibitor, was determined using a quenched fluorogenic peptide. Fluorescence was measured with a fluorescence spectrophotometer. (D) Schematic of adhesion assay and subsequent cell sorting. Fluorescently labeled SKOV3ip1 cells were mixed with 3D culture for $4 \mathrm{~h}$. The cells were mechanically removed from the plate and sorted by FACS. (E) Cell extracts were subjected to immunoblotting using an MMP-2 and MMP-9 specific antibody. The membrane was reprobed with an antibody against actin. Lane 1, unbound, floating SKOV3ip1 cells; lane 2, SKOV3ip1 cells that had attached to the 3D culture after FACS; Lane 3, 3D culture alone; Lane 4, 3D culture that had bound SKOV3ip1 cells after FACS. (F) Western blot for MT1-MMP and TIMP-2. SKOV3ip1 were plated on the 3D culture and then sorted as indicated in D. Cell extracts (top panel) or conditioned media (bottom panel) were subjected to immunoblotting with MT1-MMP or TIMP-2 antibody. HT-1080 CM was used as positive control.

stat is administered after solid tumors are established, minimal efficacy is achieved (13). Similar results have also been reported with gastric cancer and platinum-resistant OvCa xenografts $(15$, 16), indicating that efficacy of MMPI therapy is most significant when administered early in disease progression. Furthermore, in the clinical arena, it has now been reported that treatment of early-stage cancer with an MMP-2/-9 inhibitor (marimastat) might increase survival (9). We previously examined expression of MMP-2 and MMP-9 in human OvCa tissue and found that essentially all invasive OvCas, including early stage I cancers as well as metastatic implants, overexpress both MMP-2 and MMP-9, while normal ovarian tissue exhibits significantly lower levels of expres- sion $(17,18)$, indicating that MMP-2 and MMP-9 are upregulated early in OvCa progression.

Given these findings, we hypothesized that type IV collagenases, e.g., MMP-2/-9, might be important regulators for early steps of OvCa metastasis. We report here that upregulation of MMP-2 in OvCa cells is critical for their adhesion to the mesothelial lining of the peritoneum and omentum. Using an orthotopic model of OvCa cell metastasis, we found that early inhibition of MMP-2 reduced ovarian tumor cell adhesion and metastasis and thus, significantly prolonged survival of experimental mice. Subsequent or repeated treatment of solid tumors with an MMP-2 inhibitor minimally reduced ovarian tumor metastasis and had no effect on 
A 3D model

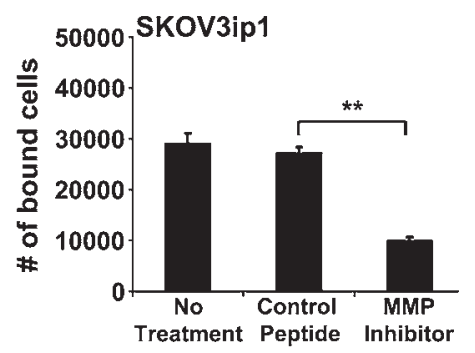

B Human omentum

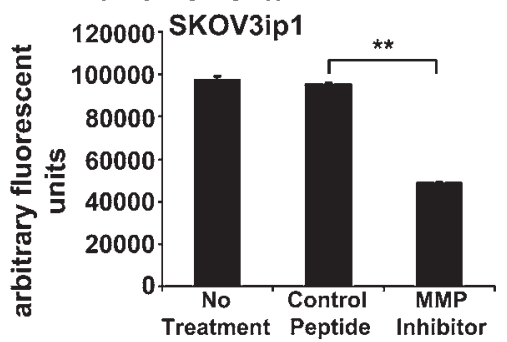

C Human peritoneum

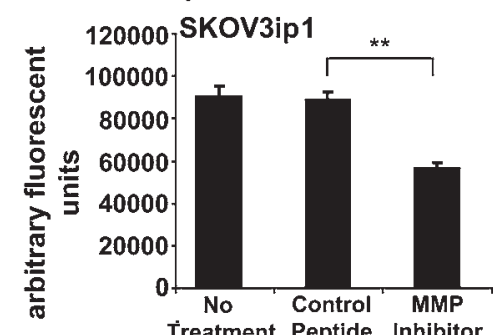

Treatment Peptide Inhibitor

In vivo-mouse

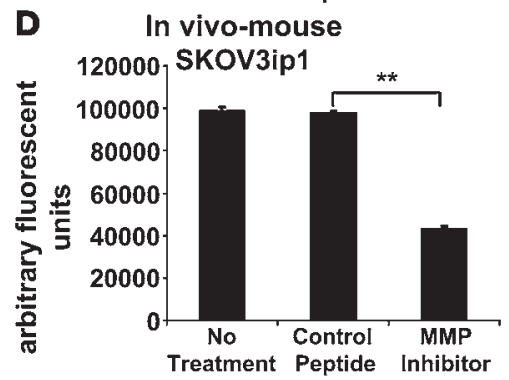

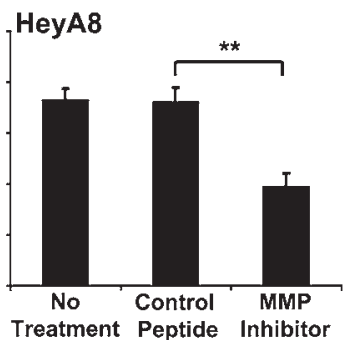
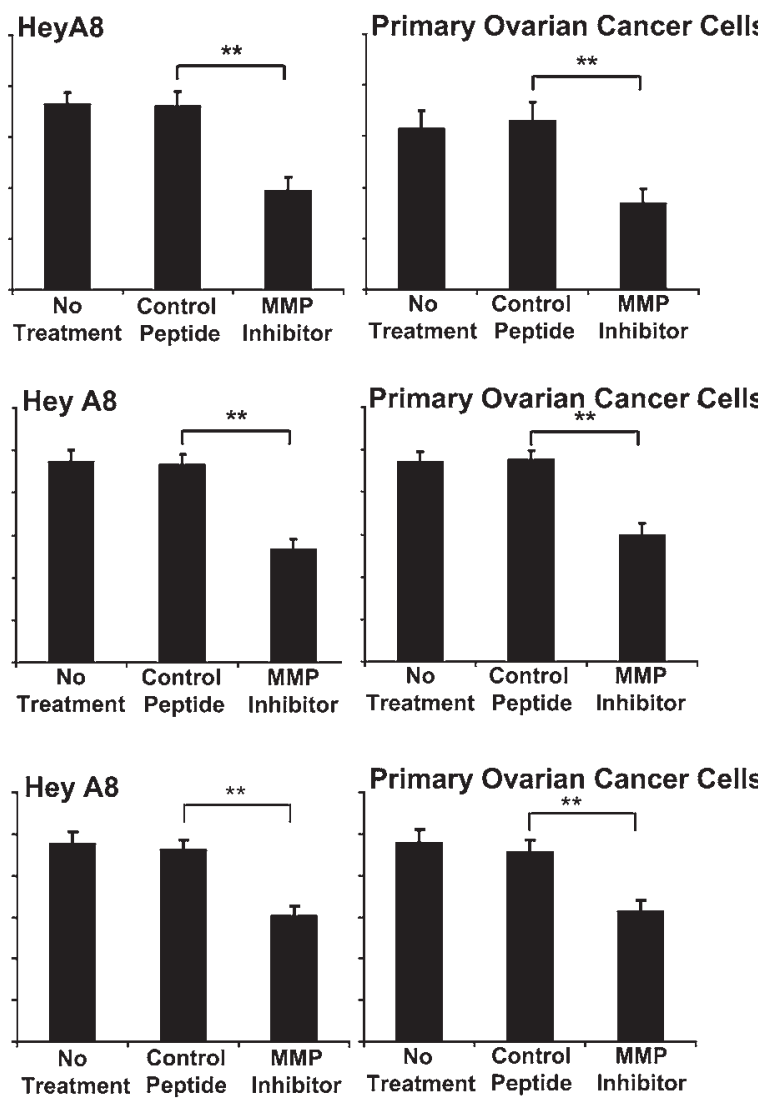

Primary Ovarian Cancer Cells

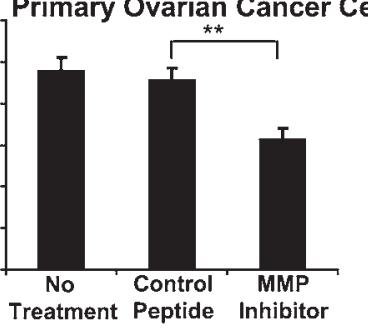

Figure 2

An MMPI inhibits adhesion of OvCa cells to a 3D culture of human omentum, full human omentum and peritoneum, and mouse omentum. SKOV3ip1, HeyA8, or primary human OvCa cells $(50,000)$ were fluorescently labeled and added to the 3D culture $(\mathbf{A})$, a piece of full human omentum (B), or full human peritoneum (C). The 3D culture, the human omentum, and the human peritoneum were washed with PBS, adherent cells on the omentum were lysed with NP-40, and fluorescence intensity was measured with a fluorescence spectrophotometer. (D) SKOV3ip1 cells were fluorescently labeled and $4 \times 10^{6}$ were cells injected i.p. into nude mice $(n=3)$. The mice were sacrificed after 4 hours, omentum and peritoneum were lysed with NP-40, and fluorescence was measured. ${ }^{\star \star} P<0.001$. Each bar represents the mean of 3 wells and SD. Each graph is representative of at least 3 independent experiments. survival. MMP-2 increased the adhesive capability of OvCa cells by specific cleavage of fibronectin (FN) and vitronectin ( $\mathrm{Vn})$, allowing for enhanced attachment of OvCa cells to FN and Vn fragments through $\alpha_{5} \beta_{1}$ and $\alpha_{V} \beta_{3}$ integrin. Therefore, our findings implicate MMP-2 in OvCa adhesion and indicate that therapeutic efficacy of MMP-2-selective inhibitors will be best achieved clinically if applied prior to peritoneal dissemination.

\section{Results}

Coculture of $\mathrm{O} v \mathrm{Ca}$ cells with mesothelial and stromal cells induces MMP-2 and MMP-9. The peritoneal cavity, including the omentum, is covered by mesothelium consisting of a confluent layer of epithelial mesothelial cells, lying juxtaposed to a layer of ECM where fibroblasts are embedded. Because peritoneal surfaces are the most common site of OvCa metastases (2), we established what we believe to be a novel organotypic $3 \mathrm{D}$ coculture model mimicking human omentum, in order to examine the role of MMP-2 and MMP-9 in adhesion (Figure 1A). Collagen I was used as the principal ECM molecule, because a dense type I collagen matrix is present in the omental and peritoneal basement membrane and because OvCa cells preferentially adhere to collagen I (19). Human primary mesothelial cells (HPMCs) and human primary fibroblasts (HPFs) were extracted from human omentum and maintained in culture for 2 passages and then evaluated for appropriate expression of cell type-specific markers in HPMCs (e.g., cytokeratin 8) and in HPFs (e.g., vimentin and proline-4hydroxylase) (20) (Figure 1A). To analyze the role of MMP-2 and MMP-9 in adhesion, the OvCa cell line, SKOV3ip1 that neither expresses nor secretes MMP-2 or MMP-9, was cocultured with fibroblasts and HPMCs, both of which secrete pro-MMP-2 and pro-MMP-9 (21). Coculture led to a significant conversion of the pro form of MMP-2 and MMP-9 to their enzymatically active forms (Figure 1B). This was paralleled by an increase in cell surface gelatinolytic activity once SKOV3ip1 cells were added to 3D cocultures (SKOV3ip1 alone, $V_{\max }$ 2,374 U/h; SKOV3ip1 cocultured, $\left.\mathrm{V}_{\max } 12,436 \mathrm{U} / \mathrm{h} ; P<0.0005\right)$. Preincubation with an MMP 


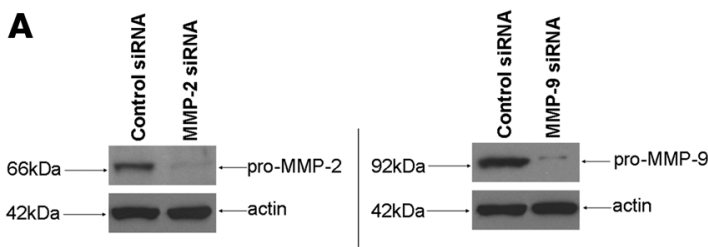

B 3D model

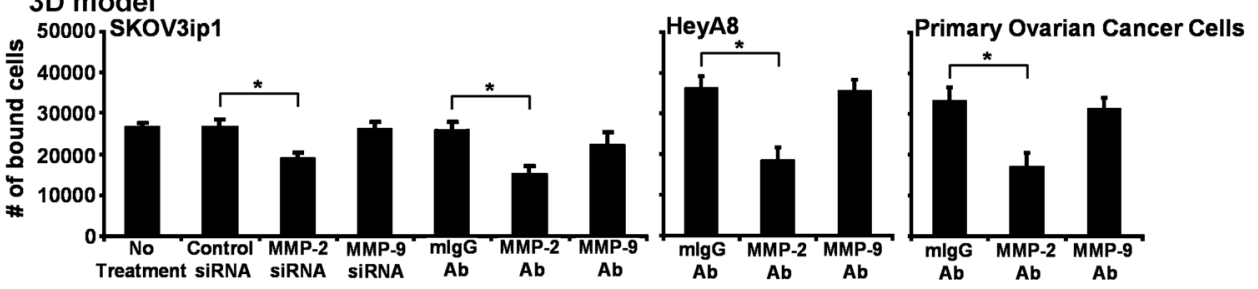

C Human omentum

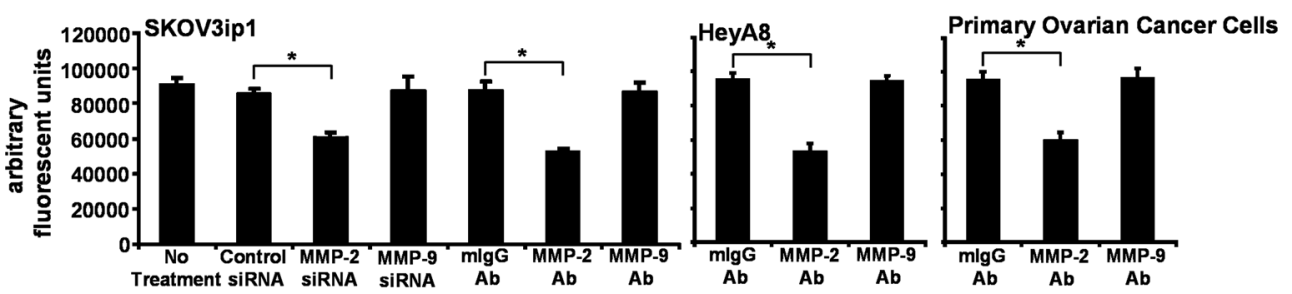

D Human peritoneum
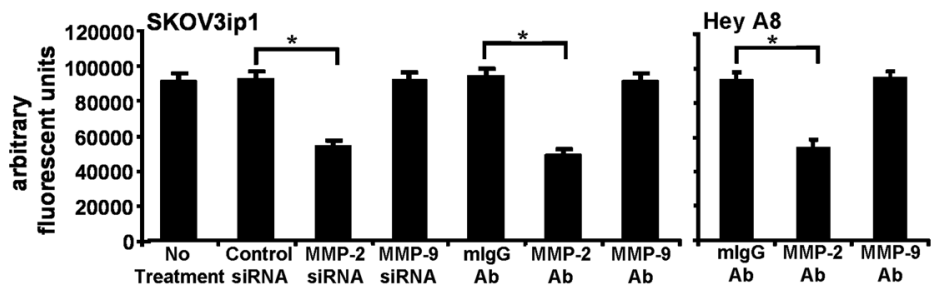

Primary Ovarian Cancer Cells

E In vivo-mouse
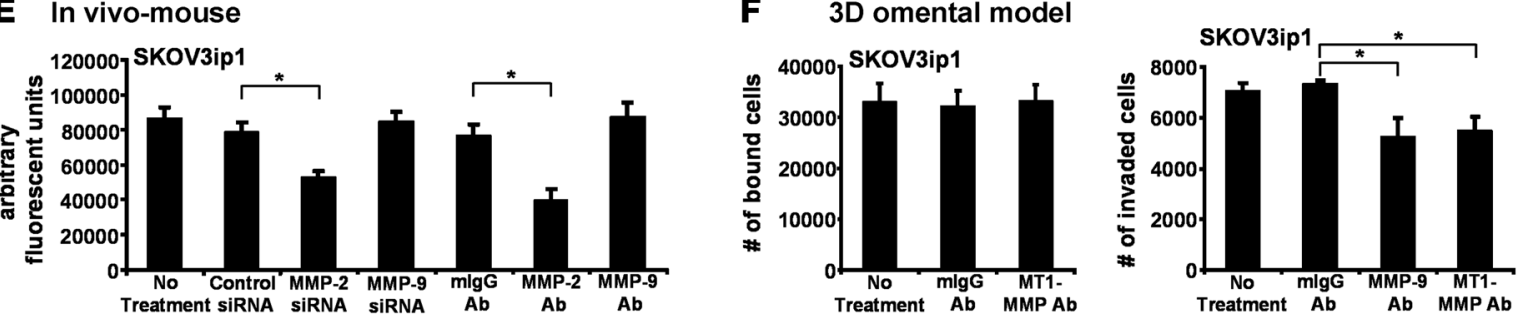

Figure 3

Attachment of OvCa cells depends on MMP-2. (A) SKOV3ip1 cells were transfected with a siRNA specific for MMP-2, MMP-9, or a control siRNA and plated on collagen type I, and MMP-2 and MMP-9 were detected by immunoblotting with specific antibodies. The membrane was reprobed with actin. (B-D) Adhesion assays. SKOV3ip1 cells were transfected with a siRNA specific for MMP-2 and MMP-9. SKOV3ip1, HeyA8, and primary OvCa cells were pretreated with an MMP-2- or MMP-9-blocking antibody and then fluorescently labeled. OvCa cells were added to the 3D culture (B), a piece of full human omentum (C), or full human peritoneum (D), and the adherent cells quantified with a fluorescence reader as described in Figure 2. (E) SKOV3ip1 cells $\left(1 \times 10^{6}\right)$ were labeled fluorescently and injected i.p. into nude mice for 4 hours $(n=3)$. Mice were sacrificed after 4 hours, omentum and peritoneum was lysed with NP-40, and fluorescence was measured. (F) Adhesion assay. SKOV3ip1 cells $\left(5 \times 10^{4}\right)$ pretreated with the MT1-MMP antibody were added to the 3D culture and an adhesion assay performed as described in Figure 2 (left panel). Invasion assay (right panel). SKOV3ip1 cells $\left(3 \times 10^{4}\right)$ were treated with a MT1-MMP- or MMP-9-blocking antibody, and invasion was analyzed after 24 hours. ${ }^{*} P<0.01$.

inhibitory peptide that binds active sites of both MMP-2 and MMP-9 with high specificity and blocks catalytic activity (22, 23) inhibited cell surface gelatinolytic activity, while the scrambled control peptide had no effect (Figure 1C), thus, implicating gelatinolytic activity during OvCa cell adhesion.

To determine which cell population was necessary for the increased presence of MMP-2/-9, SKOV3ip1 cells were fluores- cently labeled and plated on the 3D culture. Four hours later, cells were isolated by flow cytometry and immunoblotted to assess MMP-2 and MMP-9 levels (Figure 1D). Unbound SKOV3ip1 cells expressed minimal pro-MMP-2 and pro-MMP-9 protein; however, upon attachment to the $3 \mathrm{D}$ culture, both the pro and active form of MMP-2 and the pro form of MMP-9 were induced. The $3 \mathrm{D}$ culture, consisting of HPMCs and HPFs, expressed minimal 
A

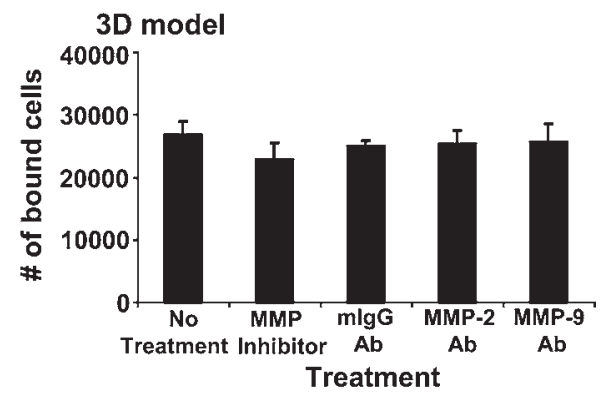

$\mathbf{B}$

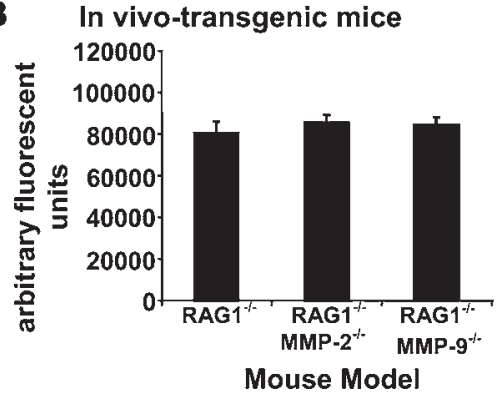

Figure 4

Host-derived MMP-2 and MMP-9 does not affect adhesion of OvCa cells. (A) The 3D culture was pretreated with either the cyclic peptide inhibiting preferentially MMP-2/-9 or a monoclonal antibody against MMP-9 or MMP-2, respectively. Subsequently, adhesion assay was performed as described in Figure 2. (B) Six-week-old RAG-1-1- mice, WT for MMP2 and MMP9 or RAG-1-/- either deficient in MMP2 (MMP2--) or MMP9 (MMP9---), were injected with fluorescently labeled SKOV3ip1. After $4 \mathrm{~h}$, the mice were sacrificed, equal parts of omentum and peritoneum were lysed in NP-40, and fluorescence was measured.

MMP-2, while a constitutively high level of MMP-9, independent of cancer cell attachment, was prominent (Figure 1E).

Matrix remodeling activity of MMP-2 is the result of a stoichiometric interaction between membrane-type 1-MMP/MMP-14 (MT1-MMP/MMP-14), a transmembrane receptor and proteolytic activator of pro-MMP-2 (3), and TIMP-2, a tissue inhibitor of metalloproteinase. Binding of SKOV3ip1 cells to the 3D culture did not alter MT1-MMP or TIMP-2 protein expression (Figure 1F). MT1-MMP was constitutively high in both the 3D culture and OvCa cells, while TIMP-2 was not secreted (Figure 1F, lower panel) or detected on the cell surface (Figure 1F, upper panel).

In view of these findings, we hypothesized that MMP-2 or MMP-9 mediated OvCa cell adhesion to mesothelium. To test this, we took a 3-pronged approach and evaluated attachment of 2 established OvCa cell lines (SKOV3ip1, HeyA8) and primary OvCa cells to the 3D coculture (Figure 2A) to full-thickness human omentum (Figure 2B) and peritoneum (Figure 2C) removed at surgery and to omentum and peritoneum within the abdominal cavity of immune-deficient mice. Incubation of SKOV3ip1, HeyA8, or primary OvCa cells with the MMP-2/-9-blocking peptide reduced cancer cell adhesion to the $3 \mathrm{D}$ coculture by $63 \%, 46 \%$, and $43 \%(P<0.005)$, respectively (Figure $2 \mathrm{~A})$. Confirming and extending these results, the MMP-2/-9 inhibiting peptide also reduced adhesion to the short-term coculture with human omentum (Figure 2B) and human peritoneum (Figure 2C). We then determined whether the peptide also inhibited in vivo adhesion of SKOV3ip1 cells to the peritoneal cavity of nude mice (Figure 2D). Adhesion was evaluated 4 hours following i.p. injection of cells, a period of time we had previously found to be required for OvCa cells to complete attachment to peritoneum and omentum (20). Untreated SKOV3ip1 cells attached to mouse peritoneum and omentum efficiently, while cells treated with the MMP-2/-9 inhibiting peptide showed a 56\% decrease $(P<0.005)$ in binding as compared with the control peptide (Figure 2D). Together, these data indicated that either MMP-2 or MMP-9 mediated OvCa cell adhesion.

Initial attachment of $\mathrm{O} v \mathrm{Ca}$ cells to the abdominal cavity is mediated by $M M P-2$. The peptide used to inhibit adhesion in Figure 2 blocks both MMP-2 and MMP-9 activity $(22,23)$. Since MMP-2 and MMP-9 possess distinct regulatory capabilities and interact with a diverse spectrum of substrates, we used specific siRNAs and blocking antibodies to MMP-2 and MMP-9 to determine which of the type IV collagenases was required for adhesion. The selective siRNAs silenced both MMP-2 and MMP-9, as demonstrated by complete loss of MMP-2 and MMP-9 protein expression, while the scrambled siRNA had no effect (Figure 3A). To determine the effect of MMP-2 or MMP-9 inhibition on adhesion, SKOV3ip1 cells transfected with the siRNA or cells pretreated with the respective antibody against MMP-2 or MMP-9 were labeled fluorescently and then incubated with the $3 \mathrm{D}$ coculture (Figure $3 \mathrm{~B}$ ), full human omentum (Figure 3C), full human peritoneum (Figure 3D), or injected i.p. into nude mice (Figure 3E). Four hours later, adhesion was measured using a fluorescence reader that detects labeled cancer cells. Both pretreatment with the MMP- 2 antibody and transfection with the MMP-2-specific siRNA significantly inhibited adhesion to the 4 different models $(P<0.005)$. This finding was not limited to SKOV3ip1 cells, since pretreatment of HeyA8, as well as several primary OvCa cells, with the MMP-2 antibody similarly inhibited adhesion to the 3D coculture, full human omentum, and peritoneum. Inhibition of MMP-9 had neither an effect on in vitro or on in vivo adhesion but did partially block invasion as previously reported (24) (Figure 3F). We ruled out the possibility that the effects identified for MMP-2 were instead mediated by MT1-MMP, since incubation of cells with a MT1-MMP neutralizing antibody (25) had no effect on OvCa cell adhesion (Figure 3F, left panel) but did block invasion (Figure 3F, right panel).

Host cells do not contribute the MMP-2 or MMP-9 necessary for peritoneal adhesion. Several studies have reported that MMP-2 and MMP-9 are expressed by activated stromal cells that thereby promote tumorigenesis (26). Moreover, in OvCa, expression of MMP-2 and MMP-9 mRNA has not only been localized to stromal cells but also to carcinoma cells (27). Therefore, we sought to determine the principal cellular source of MMP-2 and/or MMP-9 detected on OvCa cells. We found that blocking MMP-2 and MMP-9 function on HPMCs or HPFs with the MMP-2/-9 peptide or with the MMP-2 or MMP-9 antibodies did not alter adhesion of SKOV3ip1 cells (Figure 4A). Because other host-derived stromal and/or epithelial cells (e.g., adipocytes, vascular endothelial cells, leukocytes) not represented in the 3D culture might secrete MMP-2 or MMP-9 that could be used by the cancer cells in vivo, we took advantage of a genetic mouse model of MMP2 and MMP9 deficiency. Fluorescently labeled SKOV3ip1 cells were injected i.p. into RAG-1-/, RAG-1-//MMP2-/-, or RAG-1-//MMP9-/- double-deficient mice (6, 28) and their adhesion to peritoneum was evaluated (Figure 4B). We found no difference in either peritoneal or omental attachment between SKOV3ip 1 cells injected into any of the 3 experimental cohorts, indicating that MMP-2 and MMP-9 secreted 

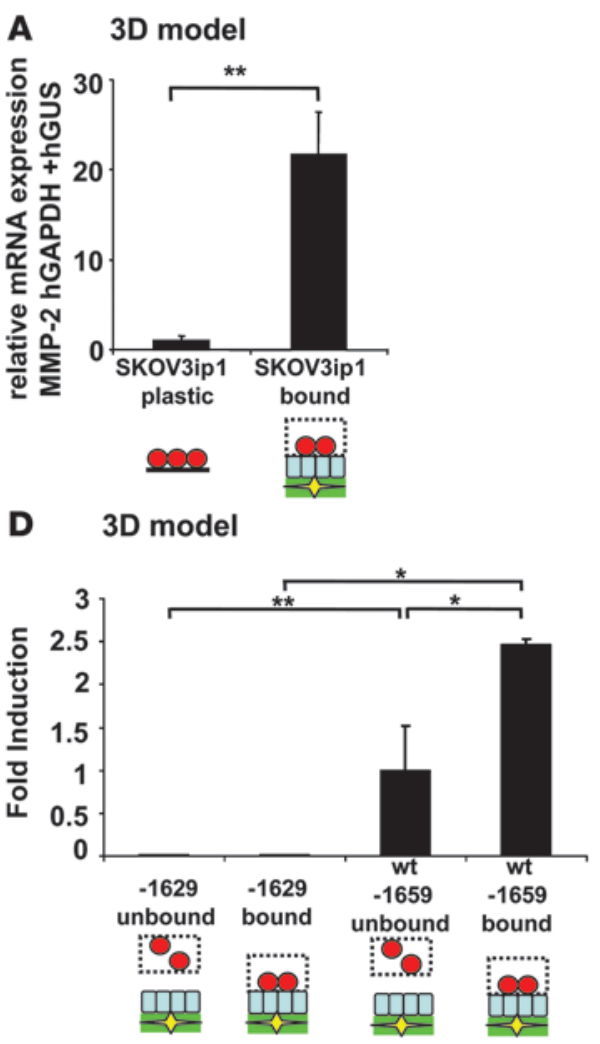
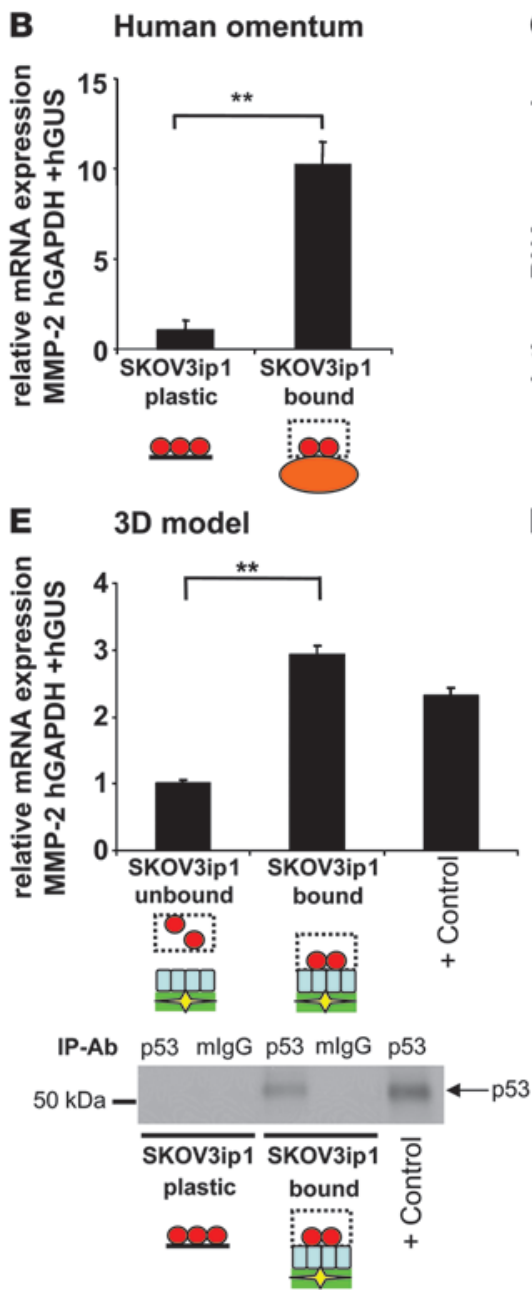

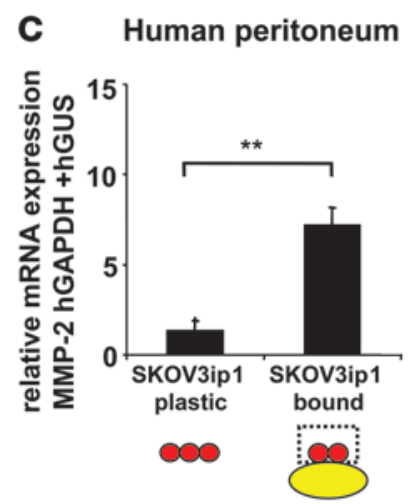

F $\quad$ 3D model

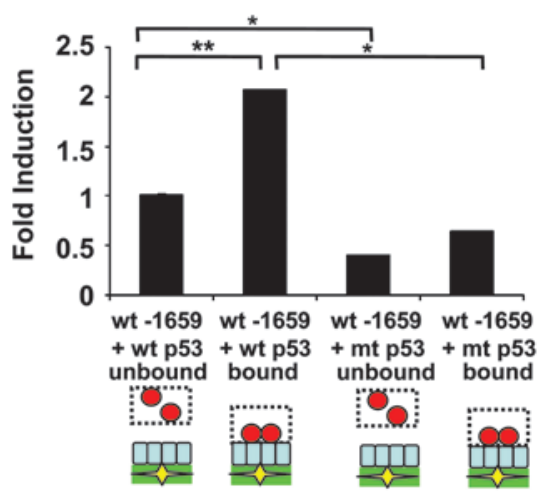

Figure 5

MMP-2 is transcriptionally upregulated in OvCa cells upon interaction with host stroma. SKOV3ip1 cells were added to the 3D culture (A), full human omentum (B), or full human peritoneum (C), and then cells were sorted by FACS. The relative expression of MMP-2 normalized to GAPDH, and huGUS was measured by TaqMan quantitative real-time RT-PCR. (D) SKOV3ip1 cells were transfected with $5 \mu \mathrm{g}$ of the $-1,659$ bp (WT) or the -1,629-bp MMP-2 promoter and adhered to 3D model, and luciferase activity was measured. (E) The relative expression of p53 normalized to GAPDH, and huGUS was measured by TaqMan quantitative real-time RT-PCR (top panel). Protein lysates from SKOV3ip1 cells cultured on plastic or 3D culture (after sorting) were immunoprecipitated with control mouse IgG or monoclonal p53 antibody, and western blot analysis for p53 was conducted on lysates using a pantropic sheep anti-p53 antibody (bottom panel). Positive control was RKO mRNA or cell lysates. (F) SKOV3ip1 cells were cotransfected with $5 \mu \mathrm{g}$ of the WT MMP-2 promoter and a WT or mutated p53 expression plasmid and adhered to the 3D model, and luciferase activity was measured. Luciferase activity was normalized to number of bound and unbound cells, and all assays were run in duplicate. ${ }^{\star} P<0.01,{ }^{* *} P<0.001$. Each graph is representative of 3 independent experiments.

by host cells was not required for attachment of OvCa cells to abdominal peritoneum or omentum.

Adhesion induces MMP-2 transcription. In view of previous reports that MMP-2 is regulated transcriptionally $(29,30)$, we investigated whether interaction of OvCa cells with host cells stimulated $M M P-2$ mRNA expression. Fluorescently labeled SKOV3ip1 cells were added to the various tissues, attached cells collected by fluorescent-activated cell sorting (FACS), and MMP-2 mRNA detected by quantitative real-time PCR. The relative expression of MMP-2 mRNA was 20-, 10-, and 7-fold higher in OvCa cells attached to the $3 \mathrm{D}$ coculture (Figure 5A), full human omentum (Figure 5B), or peritoneum (Figure $5 \mathrm{C}$ ) than in OvCa cells adhering to plastic $(P<0.00005)$. This increase in MMP-2 mRNA led us to study whether adhesion induced MMP-2 promoter activity. In a systematic search, using sequentially deleted MMP-2 promoter fragments driving expression of a luciferase reporter construct, SKOV3ip1 cells were transfected with a series of $5^{\prime}$-promoter deletion constructs and added to the 3D culture for 4 hours to allow for adhesion (Figure 5D). We found that a sequence residing from $-1,659$ bp to $-1,629$ bp was both critical for induction of $M M P-2$ promoter activation, following adhesion to the $3 \mathrm{D}$ culture, and for constitutive activity, indicating that $M M P-2 \mathrm{mRNA}$ is transcriptionally upregulated in OvCa cells by interaction with host cells (Figure $5 \mathrm{D})$. Indeed, within this 30 -bp promoter region is a consensus p53 transcription factor binding site previously found to be important for constitutive activity of MMP-2 in HT-1080 cells (29). While 
A Prevention Study

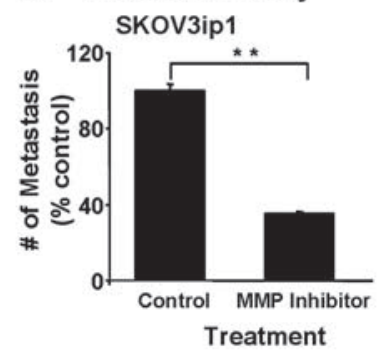

C Prevention Study

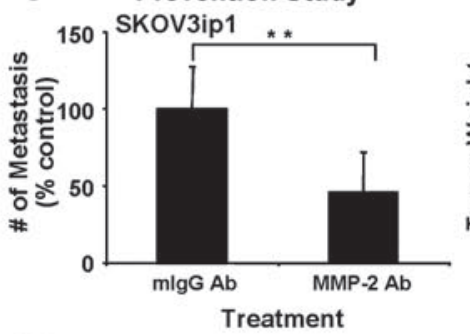

D Prevention Study

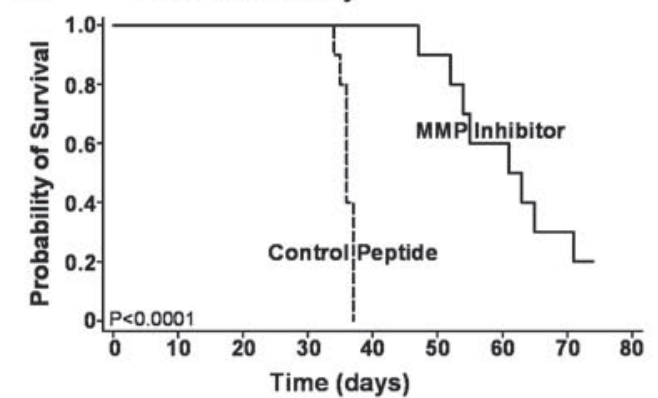

E
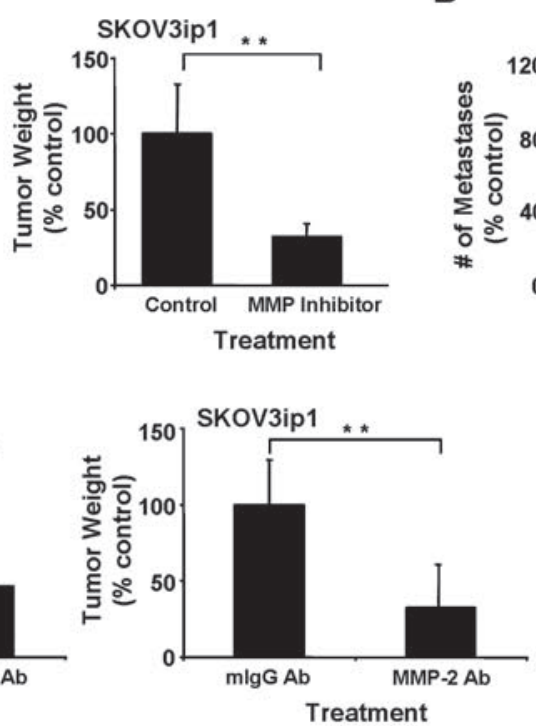

Intervention Study
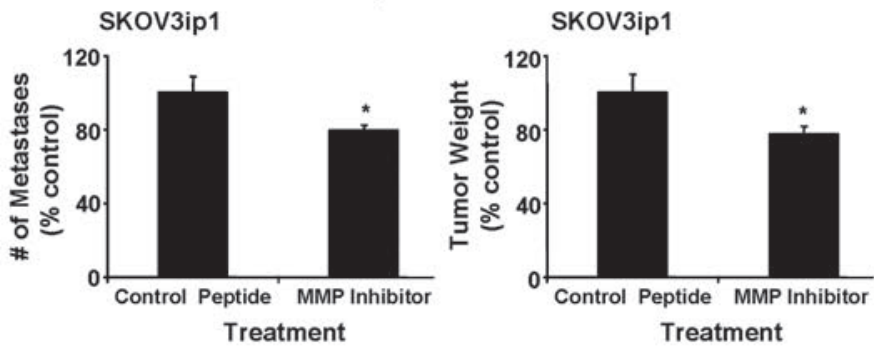

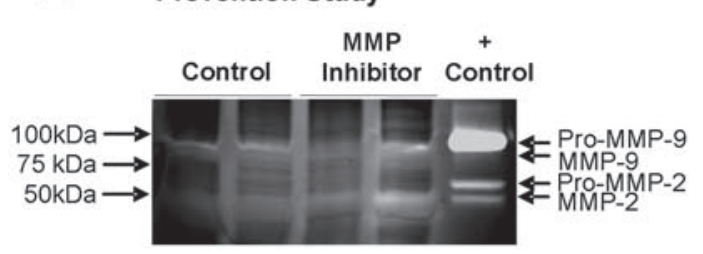

F

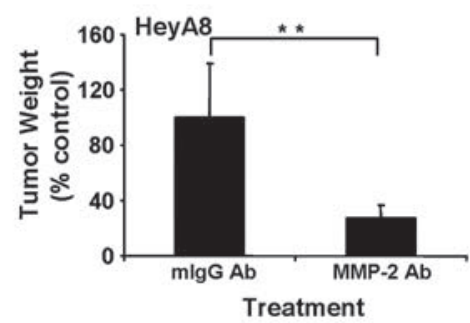

Intervention Study

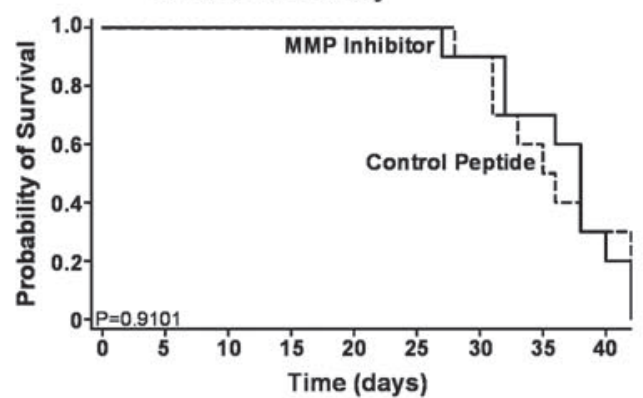

F Prevention Study

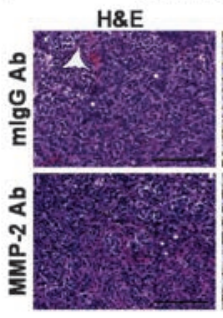

Ki67

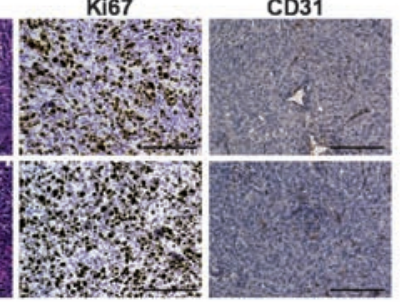

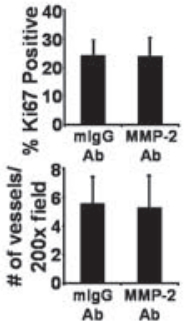

Figure 6

Single pretreatment of OvCa Cells with an MMP-2/-9 inhibitor or an MMP-2 antibody inhibits peritoneal metastases and increases survival. (A) Prevention study. SKOV3ip1 cells $\left(1 \times 10^{6}\right)$ were pretreated with MMPI or cyclic peptide and injected i.p. into nude mice. After 28 days, the number of metastasis and tumor weight were determined. ${ }^{* *} P<0.001$. (B) Intervention study. SKOV3ip1 cells $\left(1 \times 10^{6}\right)$ were injected i.p. into nude mice. After 14 days of tumor growth, mice were treated with the MMPI 3 times per week for 3 weeks. After 28 days mice were sacrificed, the number of metastasis and tumor weight were determined. The columns represent the mean and the bars the SD. ${ }^{*} P<0.01$. (C) Prevention study. SKOV3ip1 cells or HeyA8 cells $\left(1 \times 10^{6}\right)$ were pretreated with an MMP-2 antibody or the isotypic specific control antibody and then injected i.p. into nude mice. After 28 days mice were sacrificed, the number of metastasis and tumor weight were determined. ${ }^{* \star} P<0.001$. (D) Survival in prevention and intervention studies. The treatment courses of the prevention and intervention studies were conducted as described above. Mice were sacrificed once they showed signs of distress, and Kaplan-Meier curves were calculated. (E) Tumor lysates from MMPI or control peptide treated mice were subjected to gelatin zymography as described in Figure 1B. (F) Sections of tumors from MMP-2 antibody or mlgG antibody-treated mice were stained with H\&E, a Ki-67-specific antibody to detect proliferation, or a CD31-specific antibody to count microvessels. Scale bar: $100 \mu \mathrm{m}$.

there is evidence that SKOV3ip1 cells do not express p53 protein when cultured on plastic (31), our western blot and quantitative RT-PCR results showed that upon adhesion of cancer cells to the $3 \mathrm{D}$ culture, TP53 mRNA and P53 protein expression was indeed upregulated (Figure 5E). Therefore, to determine if the p53 tran- scription factor binding site plays a functional role in the adhesion-mediated regulation of MMP-2, a mutated $\mathrm{p} 53$ expression construct (32) was cotransfected with the -1,659-bp MMP-2 promoter into SKOV3ip1, which was then plated on the 3D culture. Expression of mutant p53 inhibited adhesion-mediated induction 
of the $M M P$-2 promoter (Figure $5 \mathrm{~F}$ ), indicating that induction of $M M P-2$ by adhesion is at least in part mediated through a p53 transcription factor binding site in the MMP-2 promoter.

Single pretreatment of $\mathrm{O} v \mathrm{Ca}$ cells with an MMP-2/-9 inbibitor or an $M M P-2$ antibody prevents peritoneal metastases and increases survival. Our interpretation of the data resulting from the analyses above was that MMP-2 functionally regulates adhesion of OvCa cells to the mesothelium covering the abdominal cavity. Since adhesion is the first step in OvCa metastasis, we speculated that a 1-time pretreatment (prevention study) of OvCa with an MMPI might reduce peritoneal attachment and, therefore, diminish metastasis. Indeed, after 4 weeks, mice that received an i.p. injection of SKOV3ip1 that had been treated with an MMPI developed $68 \%$ fewer metastatic tumor nodules and $65 \%$ less tumor weight than mice injected with control treated cells $(P<0.001$; Figure 6A). In contrast, mice were injected i.p. with SKOV3ip1 cells, and 2 weeks later, when their disease was established, they received repeated treatment for a total of 4 weeks (intervention study - a trial design that mimics treatment of advanced disease in humans). The mean number of metastases was reduced by only $20 \%$ and tumor weight by only $22 \%$ with the MMPI as compared with the control peptide $(P=0.007$ and $P=0.006$, respectively; Figure $6 \mathrm{~B})$. For both the tumor weight $(P=0.0001)$ and the total number of tumors $(P=0.0001)$, there was a statistically significant difference between preadministration of the inhibitor in the prevention study and repeated treatment after tumors had established in the intervention study.

Pretreatment of SKOV3ip1 with the MMP-2-blocking antibody also resulted in a significant reduction of metastatic nodules $(54 \%$; $P<0.001)$ and tumor weight $(68 \% ; P<0.001)$ as compared with an isotypic control antibody (Figure 6C). Moreover, we found that these data were not limited to a single cell line, since pretreatment of HeyA8 cells with the MMP-2 antibody yielded similar results with a $72 \%(P<0.001)$ reduction in tumor weight as compared with IgG treated controls (Figure 6C, right panel). Based on these findings, we then performed a survival study (Figure 6D), in which we found that a single pretreatment of SKOV3ip1 cells with the MMP inhibitory peptide increased median survival of inoculated mice to 63 days, whereas mice treated with the control peptide became distressed after an average of 36 days $(P<0.0005)$, a period comparable to the life span of untreated mice injected with SKOV3ip1 cells (33). In contrast, when established tumors (intervention study) were treated with the MMPI twice per week there was no statistically significant survival difference (Figure 6D).

We then analyzed tumors from the prevention studies to understand whether a single treatment with the MMPI or MMP-2 antibody affected other established MMP-2/-9 functions such as gelatinolytic activity, proliferation, apoptosis, or angiogenesis (Figure 6E). SKOV3ip1 tumors showed minimal gelatinolytic activity with no significant difference between MMPI or control treated cells (Figure 6E). The histologic appearance of tumors from the MMP-2 antibody prevention experiment was similar to that of tumors from control treated mice without evidence of necrosis or differences in apoptosis (Figure 6F and data not shown). There was also no difference in the percentage of Ki-67 positive cells in MMP-2 antibody $(24 \% \pm 7 \%)$ or mouse IgG antibody $(24 \% \pm 5.6 \%)$ treated tumors (Figure 6F) and no difference in microvessel density when staining with an antibody for CD31 $(5 \pm 2$ versus $5 \pm 2$ no. of vessels per field; Figure $6 \mathrm{~F}$ ) or detecting VEGF by western blotting (data not shown). These results indicate that MMP-2 is involved in the earliest steps of metastasis, e.g., attachment, and that once a tumor is established, inhibition of MMP-2 does not affect processes involved in later stages of metastatic growth.

MMP- 2 cleaves Vn and FN. MMP- 2 can cleave various ECM proteins (3), raising the possibility that cleavage of 1 or more ECM components into smaller fragments allows for the improved adhesion of OvCa cells to ECM. To test this hypothesis, we first confirmed the presence of $\mathrm{Vn}$ and $\mathrm{FN}$ in human omentum. Vn and FN are highly expressed in the ECM of the human omentum, and mesothelial cells produce $\mathrm{Vn}$ and FN as shown by western blot analysis (Figure 7A). $\mathrm{Vn}$ is a known substrate for MMP-2 (3) and is known to promote cancer cell migration (34). However, FN has not been previously recognized as an MMP-2 substrate. To determine if MMP-2 cleaves FN, recombinant human MMP-2 was activated with p-aminophenylmercuric acetate (APMA), then incubated with full-length FN (Figure 7B), and resolved by SDS-PAGE. Silver staining of the gel revealed several known $120-\mathrm{kDa}, 45-\mathrm{kDa}$, and 11-kDa FN fragments after incubation of FN with activated MMP-2 (Figure 7B), establishing FN as what we believe to be a novel MMP-2 target.

To test whether cleavage of $\mathrm{Vn}$ and FN by MMP-2 was of functional significance, adhesion assays were conducted on cleaved Vn or FN fragments. Indeed, significantly more SKOV3ip1 cells adhered to culture wells coated with MMP-2-cleaved Vn and FN than to wells coated with full-length $\mathrm{Vn}$ or FN alone ( $\mathrm{Vn}, 1.4 \pm 0.04$-fold increase, $P<0.0001$; FN, $1.7 \pm 0.04$-fold increase, $P<0.0001$; Figure 7C), a finding which was also confirmed in HeyA8 cells (H.A. Kenny, unpublished observations). These results were validated in a nitrocellulose filter attachment assay (35), in which full-length or MMP-2cleaved $\mathrm{FN}$ was resolved on a native Tris- $\mathrm{HCl}$ gel, transferred to nitrocellulose, and the binding of cells to filter detected by amido black cell staining (Figure 7D). OvCa cells attached to the 120- and 70-kDa MMP-2-cleaved fragment of FN, a finding that was consistent with fragments detected following MMP-2 cleavage (Figure 7B). Moreover, preincubation of cancer cells with MMP-2-cleaved Vn or FN competed with the adhesion of OvCa cells to the $3 \mathrm{D}$ culture (Vn, $1.3 \pm 0.03$-fold decrease, $P<0.01$; FN, $1.4 \pm 0.02$-fold decrease, $P<0.001$; Figure 7E). Taken together, these results indicate that MMP-2-cleaved FN and Vn promotes adhesion of OvCa cells.

Since OvCa cell adhesion is enhanced following cleavage of $\mathrm{FN}$ and $\mathrm{Vn}$, we examined the functional contribution of the FN $\left(\alpha_{5} \beta_{1}\right.$ integrin) and $V n$ receptors $\left(\alpha_{V} \beta_{3}\right.$ integrin) during the initial phase of adhesion. $\alpha_{5}$ and $\beta_{3}$ integrin were inhibited by siRNAs, and adhesion assays to the different ECM proteins performed (Figure 7F). Transfection with $\beta_{3}$ integrin siRNA significantly inhibited adhesion to MMP-2-cleaved $\operatorname{Vn}(1.7 \pm 0.03$-fold decrease; $P<0.0001)$ and FN $(1.5 \pm 0.04$-fold decrease; $P<0.0001)$ fragments (Figure 7F). Moreover, transfection with $\alpha_{5}$ integrin siRNA inhibited adhesion to MMP-2-cleaved FN fragments $(2.7 \pm 0.03$-fold decrease; $P<0.0001)$. Lastly, we assessed the inhibitory function of $\alpha_{5}, \beta_{1}$, and $\alpha_{V} \beta_{3}$ integrin-blocking antibodies (36) on OvCa cell adhesion and dependence on MMP-2 proteolytic activity. We first confirmed that treatment of OvCa cells with neutralizing antibodies against $\alpha_{5}, \beta_{1}$, and $\alpha_{V} \beta_{3}$ integrin blocked OvCa cell adhesion (Figure 7G). Then, OvCa cells were pretreated with an antibody that inhibited MMP-2 proteolytic activity, followed by treatment with the respective integrin antibody. Indeed, blocking MMP-2 abolished the inhibitory function of the $\alpha_{5}, \beta_{1}$, and $\alpha_{v} \beta_{3}$ integrin antibodies on OvCa cell adhesion. This effect was not observed when the MMP-2 antibody was substituted with isotype specific IgG and did not apply to all integrins, since the MMP-2 antibody could not abrogate the inhibitory function of a $\beta_{4}$ integrin-block- 
A

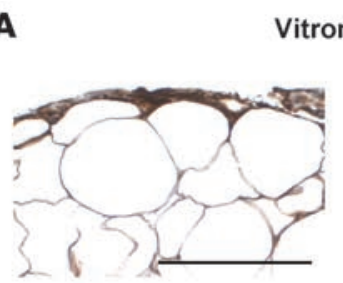

Vitronectin

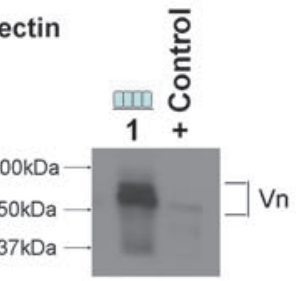

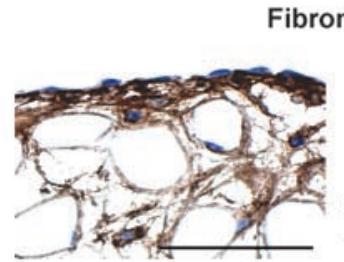

Fibronectin

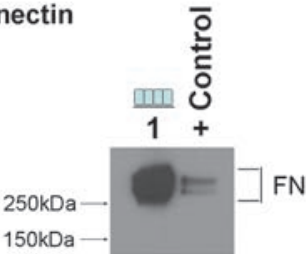

B

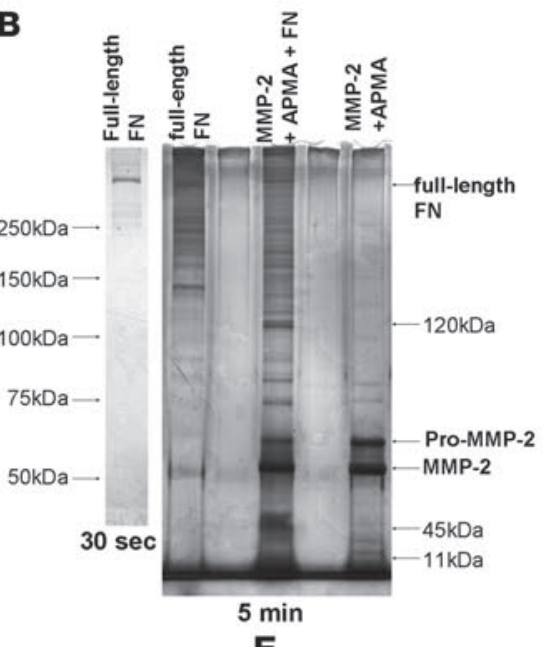

E
C
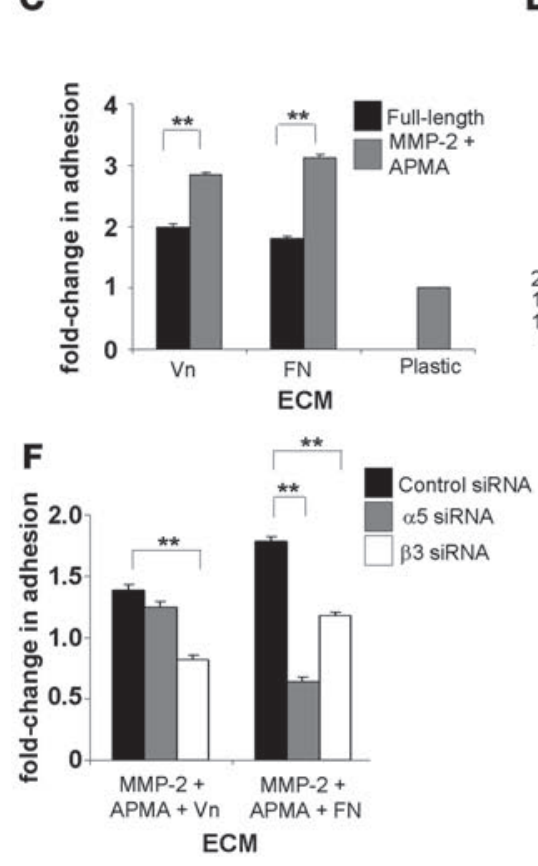

D

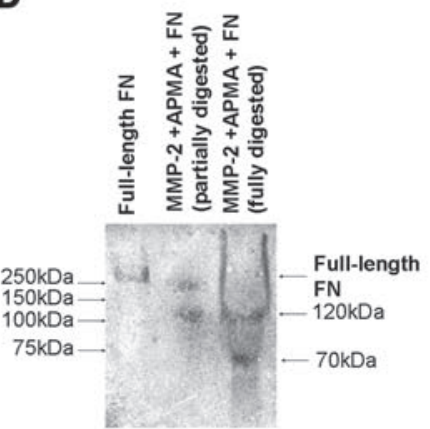

G 3D omental model
3D omental model

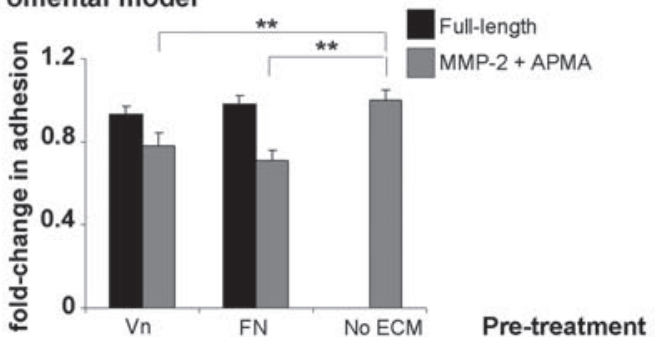

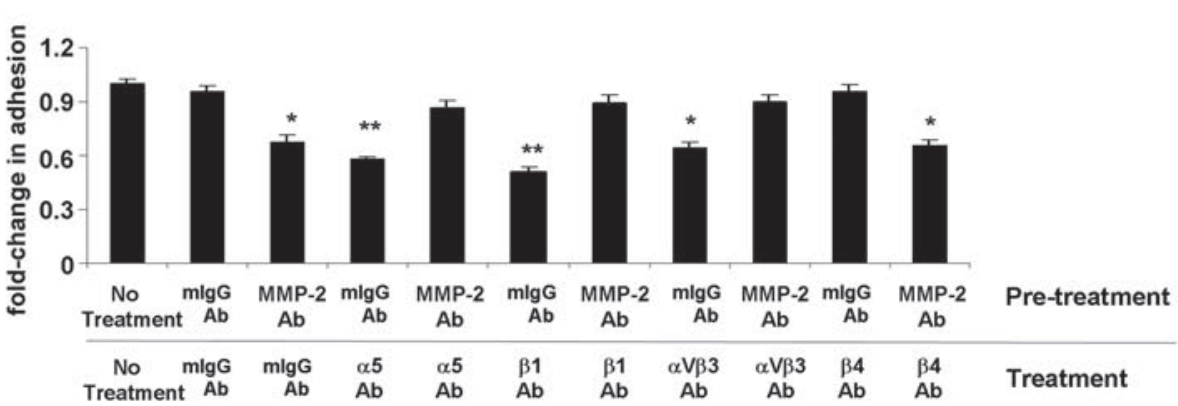

Figure 7

MMP-2 cleavage of Vn and FN increases OvCa adhesion. (A) Human omentum and peritoneum stained with Vn- or FN-specific antibodies (left panel). HPMCs were subjected to immunoblotting (right panel) using Vn- and FN-specific antibodies. Lane 1, HPMCs. HT-1080 CM was used as positive control. Original magnification, $\times 400$. (B) Full-length FN was incubated with activated MMP-2, and fragments were resolved on a $10 \%$ Tris- $\mathrm{HCl}$ gel by silver stain analysis. (C) Adhesion assay of SKOV3ip1 cells to full-length and MMP-2-cleaved Vn or FN coated plates as described in Figure 2. (D) FN and MMP-2-cleaved FN were run on a native gel and transferred to nitrocellulose. An adhesion assay was performed with $1.0 \times 10^{7}$ SKOV3ip1 cells for 4 hours, the membrane was washed, fixed, and bound cells were stained. (E) Competition assays were conducted. SKOV3ip1 cells were preincubated with full-length Vn, MMP-2-cleaved Vn, full-length FN, or MMP-2-cleaved FN, and an adhesion assay to 3D coculture was conducted. (F) SKOV3ip1 cells were transfected with a siRNA specific for $\alpha_{5}$ or $\beta_{3}$ integrin, and adhesion assay was performed to MMP-2-cleaved Vn or FN coated plates. (G) Fluorescently labeled SKOV3ip1 cells were pretreated with either an MMP-2 or a mouse isotype IgG antibody followed by treatment with $\alpha_{5}, \beta_{1}, \alpha_{v} \beta_{3}, \beta_{4}$ integrin, or mouse isotype IgG antibodies. Subsequently, an adhesion assay was performed on the 3D model. ${ }^{*} P<0.01,{ }^{* \star} P<0.001$. Each graph and picture is representative of 3 independent experiments. Scale bar: $100 \mu \mathrm{m}$.

ing antibody. Taken together, these results indicate that MMP-2 enhances binding of cancer cells through $\alpha_{V} \beta_{3}$ and $\alpha_{5} \beta_{1}$ integrin.

\section{Discussion}

Our contention that MMP-2 is a significant regulator of OvCa adhesion is supported by experiments demonstrating that a cyclic peptide preferentially inhibits MMP-2 gelatinolytic activity (22) and reduces adhesion to the 3D coculture, full human omentum/ peritoneum, and in vivo binding to mouse omentum/peritoneum (Figure 2). These findings were confirmed with an MMP-2 selective siRNA and an MMP-2-blocking antibody (Figure 3). Pretreatment of OvCa cells with either the cyclic peptide or an MMP-2 


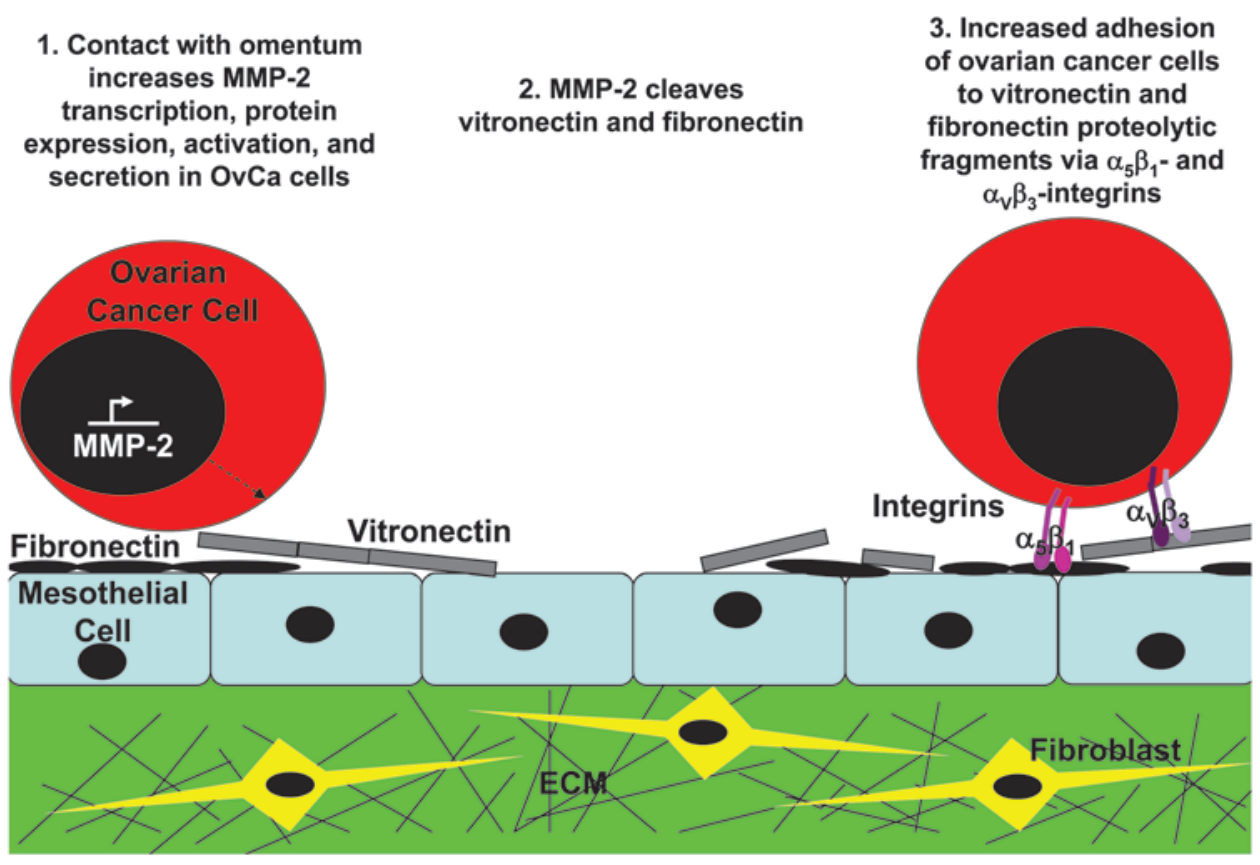

Figure 8

Proposed role of MMP-2 in early OvCa cell metastasis to the omentum and peritoneum.
The SKOV3 cell line is widely used as a p53-null cell line, and previously no p53 mRNA or protein was detected in SKOV3 or SKOV3ip1 cells when cultured on plastic $(31,40,41)$. However, only 1 study investigated the status of p53 alleles using Southern blot analysis, wherein a deletion or rearrangement of only 1 of the p53 alleles was reported (42). On the contrary, SKOV3 cells were reported as wild type for p53 in another study (43). Given that the SKOV3ip1 cell clone we used also does not express p53 on plastic but that p53 mRNA and protein are induced in the SKOV3ip1 cells upon binding to the 3D culture, we suggest that in the SKOV3ip1 cells one p53 allele is still intact (as suggested by the Southern blot in ref. 42), allowing for inducible p53 expression.

The mechanism by which MMP-2 enhances peritoneal adhesion of OvCa cells involves enzymatic neutralizing antibody abrogated cancer cell adhesion, reduced the number of metastases and tumor load, and prolonged survival in xenograft models. The enhanced efficacy of the MMPI when delivered early, prior to secondary tumor development, was not limited to OvCa. In a T cell lymphoma mouse model, treatment with a selective gelatinase inhibitor reduced liver metastasis and increased survival when the inhibitor was preincubated with the cancer cells prior to tail-vein injection but not when treatment was initiated 1 day following tumor cell inoculation (37).

We found that induction of MMP-2 in OvCa cells by normal omental fibroblasts and mesothelial cells involves direct cell-cell interaction, extending previous reports that dermal (21) and ovarian (38) fibroblasts induce MMP-2 expression in cancer cells. MMP-2 is not provided by the host, since deletion of host-derived MMP-2 or MMP-9, using RAG1/MMP-2 and MMP-9-deficient mice, did not alter OvCa cell adhesion. The specific interaction between mesothelial cells/fibroblasts and OvCa cells may explain why OvCa metastasis favors the peritoneum and omentum and rarely seeds to secondary sites that are not covered by mesothelium. In our studies, MMP-2 was induced in the cancer cells upon binding to mesothelium. Still, in situ hybridization in different cancers, including ovarian (27, 39 ), has localized MMP-2 mRNA expression to fibroblasts. However, these represent a snapshot of MMP expression at an advanced tumor stage, when the OvCa has already established. In contrast, our study examines the initial step of OvCa metastasis, when cancer cells "meet" unprepared, resting mesothelial cells and fibroblasts. In the earliest phase of metastasis, contact of mesothelium with OvCa cells induces MMP- 2 mRNA expression in the cancer cells through a consensus p53 transcription factor binding site in the MMP-2 promoter at $-1,659 \mathrm{bp}$. Consistent with this finding, we found that upon attachment of SKOV3ip1 cells to the 3D culture, $\mathrm{p} 53$ protein and mRNA expression is induced and mutation of the p53 binding site in the MMP-2 promoter abrogates induction of MMP-2. activity and cleavage of $\mathrm{FN}$ and $\mathrm{Vn}$. When a panel of integrin antibodies was tested for their ability to inhibit adhesion, $\alpha_{5} \beta_{1}$ and $\alpha_{v} \beta_{3}$ integrin-blocking antibodies inhibited adhesion, but this effect was abolished when OvCa cells were preincubated with the MMPI or an MMP-2 antibody. This finding led us to investigate the possibility that MMP-2 cleaves Vn and FN that is highly expressed in peritoneal and omental basement membranes, as well as on HPMCs. Indeed, we found that MMP- 2 cleaves FN and Vn. In addition, OvCa cells adhere more efficiently to the smaller FN and Vn cleavage products than the full-length proteins, and the increased adhesion is abrogated when $\alpha_{5} \beta_{1}$ and $\alpha_{V} \beta_{3}$ integrin are knocked down by siRNA. We propose the following general mechanism for the initial steps of OvCa adhesion. Contact of OvCa cells with mesothelium induces $M M P-2$ transcription, followed by protein expression and activation. MMP-2 then cleaves various ECM components, including $\mathrm{Vn}$ and $\mathrm{FN}$, into smaller fragments, thereby allowing for improved binding of OvCa adhesion receptors $\left(\alpha_{5} \beta_{1}\right.$ and $\alpha_{V} \beta_{3}$ integrin) to fragmented ECM (Figure 8). This model is consistent with a report demonstrating that specific cleavage of laminin- 5 by MMP-2 can induce migration of breast epithelial cells (44), and that $\alpha 3 \beta 1$ integrin on sarcoma cells selectively binds laminin-5 within exposed regions of lung vasculature (45).

We found that MMP-9 is highly expressed by mesothelial cells and fibroblasts and that OvCa cells express MMP-9 once they bind the $3 \mathrm{D}$ coculture. Nevertheless, we concluded that MMP-9 expression was not significant for initial adhesion, since we saw no inhibition of adhesion when blocking MMP-9. Nonetheless, several reports suggest that MMP-9 promotes growth and angiogenesis. Huang et al. (46) identified tumor-infiltrating macrophages as a significant source of MMP-9 and demonstrated that SKOV3ip1 cells growing in MMP-9-deficient mice are less metastatic. In a skin carcinogenesis model, MMP-9 supplied by innate immune cells is critical for tumor progression (26), tumor cell proliferation, and angiogenesis 
(47). In the RIP1-Tag2 model of pancreatic islet carcinogenesis, MMP-2 was important for tumor initiation but played no role in angiogenesis (7). These findings, together with our studies, assign unique functions to MMP-2 and MMP-9 during tumorigenesis. MMP-2 is important for adhesion, while MMP-9 exerts a functional role during angiogenesis and tumor progression.

While we remain disappointed by the failure of MMPI in clinical studies aimed at targeting late-stage disease, we are optimistic that our findings, which demonstrate that inhibition of MMP-2 during early metastasis provides a survival advantage, will encourage the reassessment of MMPI as an anticancer therapeutic agent. The MMPI, marimastat, that has selective affinity for MMP-2/-9, was evaluated in a phase III trial enrolling patients with platinum resistant, recurrent OvCa. Although the trial was not formally published, abstracts and reviews (11) reported that treatment with marimastat did not block tumor growth or improve survival, a result that is in line with other unsuccessful trials of MMPI in cancer $(8,10)$. The data presented here, demonstrating that MMP-2 is important for adhesion and early metastasis, explain at least partially, why marimastat failed in the treatment of OvCa - patients treated with marimastat had widely metastatic tumors that are less dependant on MMPs for continued growth and survival. Our prevention study shows clearly that treatment with an MMP-2 inhibitor will only exhibit efficacy if administered prior to ovarian tumor cell attachment to the peritoneal cavity. While the clinical relevance of beginning therapy before tumor inoculation is at first glance debatable, there is a subgroup of OvCa patients who might benefit from early treatment with an MMPI. These are patients whose disease at the time of surgery is limited to the ovary but have tumor cell positive ascites (FIGO stage IC to IIIA), or patients who at the end of surgery were rendered macroscopically tumor free and have only presumed "microscopic disease". Early i.p. treatment with an MMPI may reduce peritoneal attachment, although it would have less impact on the growth of cells that have already attached and have formed colonies. In summary, we have demonstrated that adhesion of OvCa cells to the peritoneal cavity is mediated, at least in part, by MMP-2 through cleavage of FN and $\mathrm{Vn}$ and that in a mouse model, pretreatment with an MMPI can reduce metastasis as well as significantly prolong survival.

\section{Methods}

Reagents. Collagen I (rat tail), FN, and Vn were purchased from BD Biosciences. Anti-vimentin, anti-prolyl-hydroxylase, anti-Ki67, and anti-CD31 antibodies were purchased from Dako Cytomation. Anti-CAM 5.2 against cytokeratin 8 was from Becton Dickinson. The $\beta_{1}$ integrin antibody was purchased from Santa Cruz, while MT1-MMP (clone AB8102), TIMP-2, $\beta_{4}$ integrin (clone ASC-3), $\alpha_{V} \beta_{3}$ integrin (clone LM609), $\alpha_{5}$ integrin (clone P1D6), and MMP-2 (clone CA-4001) antibodies were purchased from Chemicon. Anti-actin antibody was from Cell Signaling Technology. The human OvCa cell lines, SKOV3ip1 and HeyA8, were provided by Gordon B. Mills (MD Anderson Cancer Center, Houston, Texas, USA). Anti-MMP-2 and -MMP-9 (clone IM09L) monoclonal antibodies were purchased from EMD Bioscience. MMP-2/MMP-9 Inhibitor III, monoclonal anti-p53 (clone Ab-5) antibody, pantropic anti-p53 (clone Ab-7) antibody, fatty-acid free bovine serum albumin, and streptavidin-horseradish peroxidase conjugate were purchased from Calbiochem. Negative control, MMP-2, MMP-9, and $\alpha_{5}$ and $\beta_{3}$ integrin siRNA were purchased from Ambion Inc.

Animal husbandry. Mice carrying homozygous-null mutations in the MMP2 (28), MMP9 (48) and RAG-1 gene (6) were backcrossed into the FVB/n mouse strain and maintained in the homozygous-null (-/-) state. Immu- nodeficient $M M P 2^{-/-}$and $M M P 9^{-/-}$mice were generated by successive intercrossing (49). For all other experiments female athymic nude mice were used. All procedures involving animals were approved by the Institutional Committee on Animal Care, University of Chicago.

Adhesion and invasion assay. Primary OvCa cells were isolated from ascites as described (50). HPMCs and HPFs were isolated from normal human omentum, and purification was verified by vimentin, keratin 8 (CAM5.2), and prolyl-hydroxylase stainings (20). HPMCs and HPFs at early passages (passages 1-3) were used for the experiments to minimize dedifferentiation and modification of the original phenotype.

The 3D culture was assembled by embedding HPFs in collagen I and plating HPMCs as a confluent layer on top (20). Adhesion assays to the 3D culture and full human omentum or peritoneum were conducted as previously described (20). Each adhesion assays was run in triplicate. For adhesion assays to mouse peritoneum, SKOV3ip 1 cells were fluorescently labeled with CMFDA (Invitrogen), and a single-cell suspension $\left(4 \times 10^{6}\right.$ cells $/ \mathrm{ml}$ in $0.5 \mathrm{ml} \mathrm{PBS}$ ) was injected into the peritoneal cavity of athymic nude or $\mathrm{RAG1}^{-/-}$, MMP2 $2^{-/}$, or MMP-9-/ mice. After 4 hours, mice were sacrificed and full-thickness peritoneum and omentum excised. After washing to remove nonadherent cells, the tissue was lysed with $1 \%$ NP-40 and fluorescence was measured with a fluorescence spectrophotometer (Synergy HT) (33).

For the nitrocellulose adhesion assay (35), FN digested with APMAactivated MMP-2 or full-length FN was resolved on a native Tris- $\mathrm{HCl}$ gel (4\%-20\%). Proteins were transferred on nitrocellulose, the blot was washed with PBS and incubated with SKOV3ip1 cells for 4 hours. After adhesion, the blot was washed, cells were fixed with $10 \%$ formalin, stained with $0.1 \%$ amido black, and destained with a methanol/acetic acid/water solution (90:2:8). Cells bound to nitrocellulose stain dark blue. For the competition adhesion assays, SKOV3ip1 cells were preincubated with FN or cleaved FN fragments, and then an adhesion assay performed. The invasion assay was conducted for 24 hours as described (33).

Inbibition experiments. The SKOV3ip1 cells were incubated for 18 hours with MMP-2/-9 inhibitor (10 $\mu \mathrm{m})$, anti-MMP-2 $(4 \mu \mathrm{g} / \mathrm{ml})$, anti-MMP-9 $(6 \mu \mathrm{g} / \mathrm{ml})$, or anti-MT1-MMP $(12 \mu \mathrm{g} / \mathrm{ml})$ neutralizing antibody. For integrin experiments, the cells were pretreated with anti-MMP-2 or mouse IgG antibody (18 hours), followed by treatment with antibodies against $\alpha_{5}, \beta_{1}, \beta_{4}, \alpha_{V} \beta_{3}$ integrin or specific isotype mouse IgG $(10 \mu \mathrm{g} / \mathrm{ml})$. The cells were then used for in vitro or in vivo adhesion assays. MMP-2, MMP-9, $\alpha_{5}$, $\beta_{3}$ integrin, and control siRNA constructs were transfected into $2.4 \times 10^{5}$ SKOV3ip1 cells using siPORT NeoFX transfection reagent (Ambion Inc.). After culturing cells for 72 hours (optimal knockdown time), they were used in the in vitro or in vivo adhesion assays.

MMP activity measurement. Gelatinolytic zymography was performed as described with conditioned serum-free media (51).

Cell-surface-associated MMP activity was measured using a fluorogenic peptide (DQ gelatin; Invitrogen). A total of 25,000 cells were plated in a 96-well plate. The plates were incubated at $37^{\circ} \mathrm{C}$ and fluorescence was measured. Samples were run in quintuplet.

Western blot analysis. After adhesion of CMFDA-labeled SKOV3ip1 cells to the $3 \mathrm{D}$ culture, cells were sorted by FACS on a MoFlo (Dako Cytomation). This procedure separated labeled SKOV3ip1 from fibroblasts and mesothelial cells after their coculture (Figure 1D). Sorted cells were pelleted and lysed in ice-cold RIPA buffer. An equal amount $(15 \mu \mathrm{g})$ of cell extracts were separated by $10 \%$ SDS-PAGE, transferred to nitrocellulose membranes, and blocked with NET-gelatin (52). Membranes were incubated with mouse anti-MMP-2 (1:1,500), mouse anti-MMP-9 (1:1,500), rabbit anti-MT1MMP (1:1,000), mouse anti-TIMP-2 (1:500), mouse anti-actin (1:50,000), or mouse anti-FN antibody (1:2,000; soluble IST-3) overnight at $4^{\circ} \mathrm{C}$. The blots were then incubated with secondary horseradish peroxidase-conjugated IgG or IgM and visualized with enhanced chemiluminescence reagents. 
Quantitative real-time RT-PCR. After adhesion of CMFDA-labeled SKOV$3 i p 1$ cells to the $3 \mathrm{D}$ culture, cells were sorted by FACS in PBS. cDNA was synthesized after total RNA extraction using the Applied Biosystems cDNA archive kit. After RT, real-time PCR was performed using a Prism 7500 (Applied Biosystems), with predesigned and validated TaqMan probes for MMP-2, p53, GAPDH, and huGUS. The relative mRNA expression of MMP-2 or p53 was normalized to GAPDH and huGUS mRNA expression (Applied Biosystems). The reactions were run in triplicate using the following conditions: 1 cycle at $50^{\circ} \mathrm{C}$ for 2 minutes, followed by 10 minutes activation of the polymerase at $95^{\circ} \mathrm{C}$. Subsequently, 40 cycles were performed at $95^{\circ} \mathrm{C}$ for 15 seconds and $60^{\circ} \mathrm{C}$ for 60 seconds. The median Ct value was determined, and data was expressed as fold change of relative mRNA expression using the comparative Ct method (53).

Transfections. SKOV3ip1 cells were transiently transfected with the fulllength MMP-2 promoter (-1,659 bp or 5'-deletions (30) using SuperFect Transfection Reagent (QIAGEN). Eighteen hours after transfection, cells were trypsinized for adhesion assays, and the SKOV3ip1 cells $\left(1.5 \times 10^{6}\right.$ /well) were added on the $3 \mathrm{D}$ culture. After 4 hours cells were detached, lysed, and luciferase activity was analyzed (54).

Immunoprecipitation. Immunoprecipitation western blot analysis was performed on SKOV3ip1 cells that were bound to plastic or bound to 3D culture after FACs sorting as described (55).

Treatment studies. For the intervention study, SKOV3ip1 cells $\left(1 \times 10^{6}\right)$ were injected into the peritoneal cavity of nude mice. Fourteen days after injection, the control peptide ( $200 \mu \mathrm{g} /$ injection/mouse) or MMPI (200 $\mu \mathrm{g})$ was injected twice per week for 3 weeks ( 10 mice/group). For the prevention study, SKOV3ip1 cells $\left(1 \times 10^{6}\right)$, pretreated with control (PBS alone) or MMPI $(10 \mu \mathrm{M})$, were injected i.p., and 28 days after injection the mice were sacrificed (10 mice/group). The number of tumor colonies was counted and the tumor was weighed (33). For the prevention survival study, SKOV3ip 1 cells $\left(1 \times 10^{6}\right)$, pretreated for 18 hours with control peptide $(10 \mu \mathrm{m})$ or MMPI $(10 \mu \mathrm{m})$, were injected i.p. into female athymic nude mice (10 mice/group), and animals were sacrificed at the first signs of distress per institutional guidelines. For the intervention survival study, treatment was started after 14 days and continued 2 times per week for 3 weeks (10 mice/group).

Immunohistochemistry. Formalin-fixed tumors were stained with $\mathrm{H} \& \mathrm{E}$, or immunohistochemistry was performed with antibodies against Ki67 (1:100), CD31 (1:50), and FN (soluble IST-3) (Sigma-Aldrich).

$M M P-2$ cleavage of $V n$ and $F N$. Recombinant human MMP-2 (a kind gift of William Stetler-Stevenson, National Cancer Institute [NCI], Bethesda, Maryland, USA) was activated with APMA for 1 hour at $37^{\circ} \mathrm{C}$. Full-length
Vn or FN was cleaved by activated MMP- 2 for 2 hours at $37^{\circ} \mathrm{C}$. One hundred nanograms of MMP-2 and APMA-activated MMP-2 were analyzed by gelatin zymography. MMP-2 cleavage of FN were analyzed on a $10 \%$ Tris- $\mathrm{HCl}$ gel and silver stained.

Statistics. Adhesion assays were performed in triplicate, and at least 3 independent experiments were conducted. Gelatinase assays were performed in quintuplet, and at least 3 independent experiments were conducted. The mean \pm SD are reported. Significant changes were determined by 2 -sided, unpaired $t$ tests. Kaplan-Meier survival estimates were calculated to determine significant changes in prevention survival study.

\section{Acknowledgments}

We are grateful to Emily Kistner (University of Chicago) for help with the statistical analysis; Douglas Boyd (MD Anderson Cancer Center, Houston, Texas, USA) for the MMP-2 promoter constructs; Carl Maki (University of Chicago) for the p53 constructs; Anthony Montag (Department of Pathology, University of Chicago) for reading the immunohistochemical stainings; William Stetler-Stevenson for the human recombinant MMP-2; Gail Isenberg for critical review of the manuscript; Lidiya Korets (UCSF) for help with animal husbandry; and Alexandra Eichten (UCSF) for assistance with in vivo evaluation of RAG/MMP mice. E. Lengyel was supported by grants from the Gynecologic Cancer Foundation (2005-2006 GCF/Molly Cade Ovarian Cancer Research Grant), the Ovarian Cancer Research Fund (Liz Tilberis Scholars Program), and the NCI (R01 CA111882). H.A. Kenny was supported by a Penny Severns Breast, Cervical, and Ovarian Cancer Research postdoctoral fellowship from the Illinois Department of Public Health and a Graduate Training Program in Cancer Biology postdoctoral fellowship through the University of Chicago (NIH/NCI 5T32 CA09594). L.M. Coussens was supported by NIH grants CA72006, CA94168, and CA098075 and a Department of Defense Breast Cancer Research Program Era of Hope Scholar Award (BC051640).

Received for publication August 30,2007, and accepted in revised form January 30, 2008.

Address correspondence to: Ernst Lengyel, University of Chicago, Department of Obstetrics and Gynecology, 5841 South Maryland Avenue, Chicago, Illinois 60637, USA. Phone: (773) 702-6722; Fax: (773) 702-5411; E-mail: elengyel@uchicago.edu.
1. Cannistra, S.A. 2004. Cancer of the ovary. N. Engl. J. Med. 351:2519-2529.

2. Doig, T., and Monaghan, H. 2006. Sampling the omentum in ovarian neoplasia: when one block is enough. Int. J. Gynecol. Cancer. 16:36-40.

3. Egeblad, M., and Werb, Z. 2002. New functions for the matrix metalloproteinases in cancer progression. Nat. Rev. Cancer. 2:161-174.

4. Liotta, L., Abe, S., Robey, P., and Martin, G. 1979. Preferential digestion of basement membrane collagen by an enzyme derived from a metastatic murine tumor. Proc. Natl. Acad. Sci. U. S. A. 76:2268-2272.

5. Lakka, S.S., et al. 2003. Synergistic down-regulation of urokinase plasminogen activator receptor and matrix metalloproteinase-9 in SNB19 glioblastoma cells efficiently inhibits glioma cell invasion, angiogenesis and tumor growth. Cancer Res. 63:2454-2461.

6. Chantrain, C.F., et al. 2004. Stromal matrix metalloproteinase- 9 regulates the vascular architecture in neuroblastoma by promoting pericyte recruitment. Cancer Res. 64:1675-1686.
7. Bergers, G., et al. 2000. Matrix metalloproteinase-9 triggers the angiogenic switch during carcinogenesis. Nat. Cell Biol. 2:737-744.

8. Bissett, D., et al. 2005. Phase III study of matrix metalloproteinase inhibitor prinomastat in nonsmall-cell lung cancer. J. Clin. Oncol. 23:842-849.

9. Bramhall, S.R., Rosemurgy, A., Brown, P.D., Bowry, C., and Buckels, J.A.C. 2001. Marimastat as firstline therapy for patients with unresectable pancreatic cancer: a randomized trial. J. Clin. Oncol. 19:3447-3455

10. Bramhall, S.R., et al. 2002. Marimastat as maintenance therapy for patients with advanced gastric cancer: a randomised trial. Br. J. Cancer. 86:1864-1870.

11. Fletcher, L. 2000. MMPI demise spotlights target choice. Nat. Biotechnol. 18:1138-1139.

12. Coussens, L., Fingleton, B., and Matrisian, L.M. 2002. Matrix metalloproteinase inhibitors and cancer: trial and tribulations. Science. 295:2387-2392.

13. Davies, B., Brown, P.D., East, N., Crimmin, M.J., and Balkwill, F. 1993. A synthetic matrix metalloproteinase inhibitor decreases tumor burden and prolongs survival of mice bearing human ovarian carcinoma xenografts. Cancer Res. 53:2087-2091.

14. Bergers, G., Javaherian, K., Lo, K.-M., Folkman, J., and Hanahan, D. 1999. Effects of angiogenesis inhibitors on multistage carcinogenesis in mice. Science. 284:808-812.

15. Giavazzi, R., et al. 1998. Batimastat, a synthetic inhibitor of matrix metalloproteinases, potentiates the antitumor activity of cisplatin in ovarian carcinoma xenografts. Clin. Cancer Res. 4:985-992.

16. Watson, S.A., et al. 1999. Inhibition of tumor growth by marimastat in a human xenograft model of gastric cancer: relationship with levels of circulating CEA. Br. J. Cancer. 81:19-23.

17. Lengyel, E., et al. 2001. Expression of latent matrix metalloproteinase 9 (MMP-9) predicts survival in advanced ovarian cancer. Gynecol. Oncol. 82:291-298.

18. Schmalfeldt, B., et al. 2001. Increased expression of matrix metalloproteinases (MMP)-2, MMP-9, and the urokinase-type plasminogen activator is associated with progression from benign to advanced ovarian cancer. Clin. Cancer Res. 7:2396-2404. 
19. Moser, T.L., Pizzo, S.V., Bafetti, L., Fishman, D.A., and Stack, M.S. 1996. Evidence for preferential adhesion of ovarian epithelial carcinoma cells to type I collagen mediated by the $\alpha 2 \beta 1$ integrin. Int J. Cancer. 67:695-701.

20. Kenny, H.A., Krausz, T., Yamada, S.D., and Lengyel, E. 2007. Development of an organotypic peritoneal three-dimensional culture to study peritoneal attachment of ovarian cancer cells. Int. J. Cancer. 121:1463-1472.

21. Westerlund, A., Hujanen, E., Puistola, U., and Turpeenniemi-Hujanen, T. 1997. Fibroblasts stimulate human ovarian cancer cell invasion and expression of 72-kDa gelatinase A (MMP-2). Gynecol. Oncol. 67:76-82.

22. Koivunen, E., et al. 1999. Tumor targeting with a selective gelatinase inhibitor. Nat. Biotechnol. 17:768-774.

23. Hu, B., et al. 2003. Angiopoietin-2 induces human glioma invasion through the activation of matrix metalloprotease-2. Proc. Natl. Acad. Sci. U. S. A. 100:8904-8909.

24. Ramos-DeSimone, N., Moll, U., Quigley, J.P., and French, D.L. 1993. Inhibition of matrix metalloproteinase 9 activation by a specific monoclonal antibody. Hybridoma. 12:349-363.

25. Tam, E.M., Wu, Y., Butler, G., Stack, S., and Overall, C. 2002. Collagen binding properties of the membrane type-1 matrix metalloproteinase (MT1-MMP) hemopexin C domain. J. Biol. Chem. 277:39005-39014.

26. Coussens, L., Tinkle, C.L., Hanahan, D., and Werb, Z. 2000. MMP-9 supplied by bone marrow-derived cells contributes to skin carcinogenesis. Cell. 103:481-490.

27. Autio-Harmainen, H., et al. 1993. Expression of 72 kilodalton type IV collagenase (gelatinase A) in benign and malignant ovarian tumors. Lab. Invest. 69:312-321.

28. Itoh, T., et al. 1998. Reduced angiogenesis and tumor progression in gelatinase A-deficient mice. Cancer Res. 58:1048-1051.

29. Yan, C., Wang, H., and Boyd, D. 2002. ATF3 represses 72-kDa type IV collagenase (MMP-2) expression by antagonizing p53- dependent transactivation of the collagenase promoter. J. Biol. Chem. 277:10804-10812.
30. Bian, J., and Sun, Y. 1997. Transcriptional activation by p53 of the human type IV collagenase (gelatinase A or matrix metalloproteinase 2) promoter. Mol. Cell. Biol. 17:6330-6338.

31. Modesitt, S.C., et al. 2001. In vitro and in vivo adenovirus-mediated $\mathrm{p} 53$ and $\mathrm{p} 16$ tumor supressor therapy in ovarian cancer. Clin. Cancer Res. 7:1765-1772

32. Pletenpol, J.A., et al. 1994. Sequence-specific transcriptional activation is essential for growth supression by p53. Proc. Natl. Acad. Sci. U. S. A. 91:1998-2002.

33. Sawada, K., et al. 2007. C-Met overexpression is a prognostic factor in ovarian cancer and an effective target for inhibition of peritoneal dissemination and invasion. Cancer Res. 67:1670-1680.

34. Carreiras, F., et al. 1999. Migration properties of the human ovarian adenocarcinoma cell line IGROV1: importance of $\alpha v \beta 3$ integrins and vitronectin. Int. J. Cancer. 80:285-294.

35. Pierschbacher, M.D., Hayman, E.G., and Ruoslahti, E. 1985. The cell attachment determinant in fibronectin. J. Cell. Biochem. 28:115-126.

36. Cannistra, S.A., Ottensmeier, C., Niloff, J., Orta, B., and DiCarlo, J. 1995. Expression and function of $\beta 1$ and $\alpha v \beta 3$ integrins in ovarian cancer. Gynecol. Oncol. 58:216-225.

37. Krüger, A., et al. 2005. Antimetastatic activity of a novel mechanism-based gelatinase inhibitor. Cancer Res. 65:3523-3526.

38. Boyd, R., and Balkwill, F. 1999. MMP-2 release and activation in ovarian carcinoma: the role of fibroblasts. Br. J. Cancer. 80:315-321.

39. Naylor, M.S., Stamp, G.W., Davies, B.D., and Balkwill, F.R. 1994. Expression and activity of MMPs and their regulators in ovarian cancer. Int. J. Cancer. 58:50-56.

40. Vikhanskaya, F., Erba, E., D'Incalci, M., and Broggini, M. 1994. Introduction of wild-type p53 in a human ovarian cancer cell line not expressing endogenous p53. Nucleic Acids Res. 22:1012-1017.

41. Brader, K.R., et al. 1997. Adenovirus E1A expression enhances the sensitivity of an ovarian cancer cell line to multiple cytotoxic agents through an apoptotic mechanism. Clin. Cancer Res. 3:2017-2024.

42. Yaginuma, Y., and Westphal, H. 1992. Abnormal structure and expression of the p53 gene in human ovarian carcinoma cell lines. Cancer Res. 52:4196-4199.

43. Hagiwara, K., McMenamin, M.G., and Harris, C.C. 1999. Mutational analysis of the $\mathrm{p} 63 / \mathrm{p} 73 \mathrm{~L} / \mathrm{p} 51 /$ p40/CUSP/Ket gene in human cancer cell lines using intronic primers. Cancer Res. 59:4165-4169.

44. Giannelli, G., et al. 1997. Induction of cell migration by matrix metalloprotease- 2 cleavage of laminin-5. Science. 277:225-228.

45. Wang, H., et al. 2004. Tumor cell $\alpha 3 \beta 1$ integrin and vascular laminin-5 mediate pulmonary arrest and metastasis. J. Cell Biol. 164:935-941.

46. Huang, S., et al. 2002. Contributions of stromal metalloproteinase- 9 to angiogenesis and growth of human ovarian carcinoma in mice. J. Natl. Cancer Inst. 94:1134-1142.

47. Coussens, L., et al. 1999. Inflammatory mast cells upregulate angiogenesis during squamous epithelial carcinogenesis. Genes Dev. 13:1382-1397.

48. Vu, T.H., et al. 1998. MMP-9/gelatinase B is a key regulator of growth plate angiogenesis and apoptosis of hypertrophic chondrocytes. Cell. 93:411-422.

49. Mombaerts, P., et al. 1992. RAG-1 deficient mice have no mature B and T lymphocytes. Cell. 68:869-877.

50. Lounis, H., et al. 1994. Primary cultures of normal and tumoral human ovarian epithelium: a powerful tool for basic molecular studies. Exp. Cell Res. 215:303-309.

51. Lengyel, E., et al. 1995. Induction of Mr 92,000 type IV collagenase expression in a squamous cell carcinoma cell line by fibroblasts. Cancer Res. 55:963-967.

52. Ried, S., et al. 1999. Activation mechanisms of the urokinase-type plasminogen activator promoter by hepatocyte growth factor/scatter factor (HGF/SF). J. Biol. Chem. 274:16377-16386.

53. Pfaffl, M., et al. 2001. A new mathematical model for relative quantification in real-time RT-PCR. Nucleic Acids Res. 29:e45.

54. Hapke, S., et al. 2001. $\beta_{3}$ A-integrin downregulates the urokinase-type plasminogen activator receptor (u-PAR) through a PEA3/ets transcriptional silencing element in the u-PAR promoter. Mol. Cell. Biol. 21:2118-2132.

55. Nie, L., Sasaki, M., and Maki, C.G. 2007. Regulation of p53 nuclear export through sequential changes in conformation and ubiquitination. J. Biol. Chem. 282:14616-14625. 